ITEP/TH-27/05

\title{
Matrix models with hard walls: Geometry and solutions
}

\author{
L. Chekhov ${ }^{\mathrm{a}}$ \\ Steklov Mathematical Institute, Moscow, Russia \\ Institute for Theoretical and Experimental Physics, Moscow, Russia, \\ Poncelet Laboratoire International Franco-Russe, Moscow, Russia, \\ and Department of Mathematics and Statistics, Concordia University, Montreal, Canada
}

\begin{abstract}
We discuss various aspects of most general multisupport solutions to matrix models in the presence of hard walls, i.e., in the case where the eigenvalue support is confined to subdomains of the real axis. The structure of the solution at the leading order is described by semiclassical, or generalized Whitham-Krichever hierarchies as in the unrestricted case. Derivatives of tau-functions for these solutions are associated with families of Riemann surfaces (with possible double points) and satisfy the Witten-Dijkgraaf-Verlinde-Verlinde equations. We then develop the diagrammatic technique for finding free energy of this model in all orders of the 't Hooft expansion in the reciprocal matrix size generalizing the Feynman diagrammatic technique for the Hermitian one-matrix model due to Eynard.
\end{abstract}

The matrix models and their so-called multisupport (multicut) solutions became important again after the studies in $\mathcal{N}=1$ SUSY gauge theories [1], 2] and due to Dijkgraaf and Vafa [3, who proposed to calculate the low energy superpotentials using the partition function of multicut solutions. These solutions, known since early 90th (see, e.g., 4, 5]), were revived by Bonnet, David and Eynard [6.

Dijkgraaf and Vafa proposed to consider the nonperturbative superpotentials of $\mathcal{N}=1$ SUSY gauge theories in four dimensions arising from the partition functions of the one-matrix model (1MM) in the leading order in $1 / N, N$ being the matrix size. The leading order (of the 't Hooft $1 / N$-expansion) of the matrix model is described by the semiclassical tau-function of the so-called universal WhithamKrichever (WK) hierarchy [7] (see also [8, 9, 10; the details about one-matrix and two-matrix cases see in [12] and [13]). At the same time, matrix integrals beyond large- $N$ limit are tau-functions of the

${ }^{\mathrm{a} E-m a i l: ~ c h e k h o v @ m i . r a s . r u ~}$ 
hierarchies of integrable equations of the KP/Toda type 14. For the planar single-cut solutions, the matrix-model partition functions become $\tau$-functions of the dispersionless Toda hierarchy, one of the simplest example of the WK hierarchy.

One may also consider more general solutions to matrix models, identifying them with generic solutions to the loop (Schwinger-Dyson, or Virasoro) equations [15], or the Ward identities satisfied by matrix integrals [16]. We discuss an interesting class of multi-cut, or multi-support, solutions to the loop equations possessing multi-matrix integral representations [6, 17, 18, 19. These solutions are associated with families of Riemann surfaces and form a sort of a basis in the space of all solutions to the loop equations [18, 19] (like the finite-gap solutions form a similar basis in the space of all solutions to an integrable hierarchy). They can be distinguished by their "isomonodromic" properties - switching on higher matrix model couplings or $1 / N$-corrections does not change the family of Riemann surfaces, but just reparameterizes the moduli as functions of these couplings. This property is directly related to that the partition functions of these solutions are semiclassical tau-functions (also called the prepotentials of the corresponding Seiberg-Witten-like systems).

The geometrical properties of these solutions were extensively studied in [20, 21], and the comprehensive procedure for calculating $1 / N$-expansion terms was developed in [22, 23]. In this paper, we give the review of methods of these papers and extend them to the case of matrix models with hard walls (hard edges). Those are models in which we introduce rigid restrictions on the domain of admissible eigenvalues of the potential. It is well-known that the behavior of the eigenvalue distribution changes dramatically in the presence of such walls: it has inverse square-root singularity as approaching the wall (see, e.g., Szegø 24]).

Our aim in this paper is to show how the solutions with hard walls can be nicely incorporated in the general setting of solutions to 1MM. Actually we show that all the basic ingredients of 1MM solutions, that is, the WK hierarchy, the WDVV equations, and the diagrammatic technique for evaluating corrections in $1 / N$, turn out to be common for solutions with and without hard walls and only the hyperelliptic Riemann surface representing the spectral curve is changed. Technical solutions however apparently develop distinctions, which we indicate in what follows.

Recently, models with hard walls in the planar limit of two-matrix models were constructed [25, 26].

For the generality of presentation, we use the term 1MM to denote a Hermitian one-matrix model possibly with hard walls.

In Sec. 11 we describe the general properties of multi-cut solutions of matrix models. We derive the basic set of constraints on the matrix model partition functions and present them in the form of the master loop equation.

Before presenting the general procedure of solving the master loop equation order by order to find the loop mean (one-point resolvents) of the matrix model potential, we present the special investigation of just the leading (planar) approximation. We prove that the free energy of the 1MM in the planar $($ large- $N$ ) limit coincides with the prepotential of a Seiberg-Witten-Whitham-Krichever theory. 
The corresponding WK hierarchy is formulated in terms of Abelian differentials on (a family of) Riemann surfaces. This implies that the main quantities in matrix models can be expressed in geometrical terms and allow calculating derivatives of the matrix model free energy. Indeed, we demonstrate in Sec. 2 that the second derivatives of the 1MM free energy can be expressed through the so-called Bergmann bi-differential. On this language we derive the general relation satisfied by the matrix model potentials when differentiating w.r.t. Whitham times. We then come to third derivatives. Indeed, it is typical (but not compulsory) for WK tau-functions to satisfy a set of (generalized) Witten-Dijkgraaf-Verlinde-Verlinde (WDVV) equations [27, 28, 29] that are differential equations with respect to Whitham times involving third derivatives. These equations are usually considered an evidence for an underlying topological theory. In Sec. 2] we prove that the semiclassical tau-function of the multi-support solutions to matrix models satisfies the WDVV equations in the case of general $1 \mathrm{MM}$ solution, i.e., in the case of arbitrary number of nonzero canonical times, which include the times of the potential and the occupation numbers (filling fractions), which indicate the portions of eigenvalues that dwell on the related intervals of the eigenvalue support. The hard-wall parameters, although being out of the set of canonical variables, affect nevertheless the dimension of the set of nondegenerate WDVV equations. This completes an interpretation of the results of [1, 2] in terms of semiclassical hierarchies. The WDVV equations follow from the residue formula and associativity of some algebra (e.g., of the holomorphic differentials on the Riemann surface) 29, 30. We present the proof of the residue formula and extra conditions following [20. Analogous results hold true in the two-matrix model 31]

In Sec. 3] we describe the diagrammatic procedure that allows evaluating order by order all the corrections in $1 / N$ in the $1 \mathrm{MM}$ with hard walls. In the same section we describe the procedure of [23] to integrate the obtained answer to obtain the expression for the free energy itself. This procedure works in all genera except the subleading (torus) approximation, which, correspondingly, deserves the special investigation (as in the case of the standard 1MM). We therefore devote the special section 4 to the calculation of the free-energy subleading correction. Note again that analogous formulas were obtained in the two-matrix model case by Eynard, Kokotov, and Korotkin [32.

\section{Matrix models and Riemann surfaces}

\subsection{Matrix integrals and resolvents}

Consider the $1 \mathrm{MM}$ integral

$$
\int_{N \times N ; E} D X e^{-\frac{1}{\hbar} \operatorname{tr} V(X)}=e^{\frac{1}{\hbar^{2}} \mathcal{F}} \equiv \mathcal{Z}
$$

where $V(X)=\sum_{k \geq 1} t_{k} X^{k}, \hbar=\frac{t_{0}}{N}$ is a formal expansion parameter, the integration goes over the $N \times N$ matrices, $D \bar{X} \propto \prod_{i<j} d \Re X_{i j} d \operatorname{Im} X_{i j} \prod_{i} d \Re X_{i i}$, and we also assume that the integration domain $E$ for the eigenvalues of the matrix $X$ comprises a number of (possibly half-infinite) intervals, $E=$ 
$\cup_{\beta=1}^{q / 2}\left[a_{2 \beta-1}, a_{2 \beta}\right]$. The topological expansion of the Feynman diagrams series is then equivalent to the expansion in even powers of $\hbar$ for

$$
\mathcal{F} \equiv \mathcal{F}\left(\hbar, t_{0}, t_{1}, t_{2}, \ldots\right)=\sum_{h=0}^{\infty} \hbar^{2 h} \mathcal{F}_{h}
$$

Customarily $t_{0}=\hbar N$ is the scaled number of eigenvalues. We assume the potential $V(\lambda)$ to be a polynomial of the fixed degree $m+1$, with the fixed constant "highest" time $t_{m+1}=1$.

The averages, corresponding to the partition function (11) are defined as usual:

$$
\langle f(X)\rangle=\frac{1}{\mathcal{Z}} \int_{N \times N ; E} D X f(X) \exp \left(-\frac{1}{\hbar} \operatorname{tr} V(X)\right)
$$

and it is convenient to use their generating functionals: the one-point resolvent

$$
W(\lambda)=\hbar \sum_{k=0}^{\infty} \frac{\left\langle\operatorname{tr} X^{k}\right\rangle}{\lambda^{k+1}}
$$

as well as the $s$-point resolvents $(s \geq 2)$

$$
W\left(\lambda_{1}, \ldots, \lambda_{s}\right)=\hbar^{2-s} \sum_{k_{1}, \ldots, k_{s}=1}^{\infty} \frac{\left\langle\operatorname{tr} X^{k_{1}} \cdots \operatorname{tr} X^{k_{s}}\right\rangle_{\mathrm{conn}}}{\lambda_{1}^{k_{1}+1} \cdots \lambda_{s}^{k_{s}+1}}=\hbar^{2-s}\left\langle\operatorname{tr} \frac{1}{\lambda_{1}-X} \cdots \operatorname{tr} \frac{1}{\lambda_{s}-X}\right\rangle_{\text {conn }}
$$

where the subscript "conn" pertains to the connected part.

These resolvents are obtained from the free energy $\mathcal{F}$ through the action

$$
\begin{aligned}
W\left(\lambda_{1}, \ldots, \lambda_{s}\right) & =\hbar^{2} \frac{\partial}{\partial V\left(\lambda_{s}\right)} \frac{\partial}{\partial V\left(\lambda_{s-1}\right)} \cdots \frac{\partial \mathcal{F}}{\partial V\left(\lambda_{1}\right)}= \\
& =\frac{\partial}{\partial V\left(\lambda_{s}\right)} \frac{\partial}{\partial V\left(\lambda_{s-1}\right)} \cdots \frac{\partial}{\partial V\left(\lambda_{2}\right)} W\left(\lambda_{1}\right),
\end{aligned}
$$

of the loop insertion operator

$$
\frac{\partial}{\partial V(\lambda)} \equiv-\sum_{j=1}^{\infty} \frac{1}{\lambda^{j+1}} \frac{\partial}{\partial t_{j}}
$$

Therefore, if one knows exactly the one-point resolvent for arbitrary potential, all multi-point resolvents can be calculated by induction. In the above normalization, the genus expansion has the form

$$
W\left(\lambda_{1}, \ldots, \lambda_{s}\right)=\sum_{h=0}^{\infty} \hbar^{2 h} W_{h}\left(\lambda_{1}, \ldots, \lambda_{s}\right), \quad s \geq 1
$$

which is analogous to genus expansion (2). 


\subsection{Master loop equation}

We begin with deriving conditions on integral (11) coming from its invariance w.r.t. changing the integration variables. For this, we first to the eigenvalue representation assuming

$$
e^{\frac{1}{\hbar^{2}} \mathcal{F}}=\int_{E\left(\left\{a_{\beta}\right\}\right)} D X e^{-\frac{1}{\hbar} \operatorname{tr} V(X)} \simeq \int_{E\left(\left\{a_{\beta}\right\}\right)} \prod_{i=1}^{t_{0} / \hbar} d \lambda_{i} \Delta^{2}(\lambda) e^{-\frac{1}{\hbar} \sum_{i=1}^{t_{0} / \hbar} V\left(\lambda_{i}\right)}
$$

up to the volume of the unitary group $U\left(t_{0} / \hbar\right)$. Here, as usual, we let $\Delta(\lambda)$ denote the Vandermonde determinant of variables $\lambda_{i}$. In the latter expression, we perform the change of variables

$$
\lambda_{i}=\tilde{\lambda}_{i}+\varepsilon \tilde{\lambda}_{i}^{p+1}, \quad p \geq-1,
$$

obtaining

$$
e^{\frac{1}{\hbar^{2}} \mathcal{F}}=\int_{E\left(\left\{a_{\beta}-\varepsilon a_{\beta}^{p+1}\right\}\right)} \prod_{i=1}^{t_{0} / \hbar} d\left(\tilde{\lambda}_{i}+\varepsilon \tilde{\lambda}_{i}^{p+1}\right) \Delta^{2}\left(\tilde{\lambda}+\varepsilon \tilde{\lambda}^{p+1}\right) e^{-\frac{1}{\hbar} \sum_{i=1}^{t_{0} / \hbar} V\left(\tilde{\lambda}_{i}+\varepsilon \tilde{\lambda}_{i}^{p+1}\right)} .
$$

Segregating the coefficient by $\varepsilon$ in a standard way and equating it to zero, we obtain the Virasoro conditions to be satisfied by integral (11) (see [33])

$$
\left(-\sum_{\beta=1}^{q} a_{\beta}^{p+1} \frac{\partial}{\partial a_{\beta}}+\sum_{k=0}^{p} \hbar^{2} \frac{\partial}{\partial t_{k}} \cdot \frac{\partial}{\partial t_{p-k}}+\sum_{k=1}^{\infty} k t_{k} \frac{\partial}{\partial t_{k+p}}\right) \mathcal{Z}=0, \quad p \geq-1,
$$

where by definition

$$
\frac{\partial}{\partial t_{0}} \mathcal{Z} \equiv-\frac{t_{0}}{\hbar^{2}} \mathcal{Z}, \text { or } \frac{\partial}{\partial t_{0}} \mathcal{F} \equiv-t_{0}
$$

In terms of $\mathcal{F}$, Eqs. (11) become

$$
-\sum_{\beta=1}^{q} a_{\beta}^{p+1} \frac{\partial \mathcal{F}}{\partial a_{\beta}}+\sum_{k=0}^{p} \frac{\partial \mathcal{F}}{\partial t_{k}} \frac{\partial \mathcal{F}}{\partial t_{p-k}}+\hbar^{2} \sum_{k=0}^{p} \frac{\partial^{2} \mathcal{F}}{\partial t_{k} \partial t_{p-k}}+\sum_{k=1}^{\infty} k t_{k} \frac{\partial \mathcal{F}}{\partial t_{k+p}}=0, \quad p \geq-1,
$$

and contracting these Virasoro conditions with $\lambda^{-p-2}$, we obtain the master loop equations [15] of the $1 \mathrm{MM}$ with hard walls [16]:

$$
\sum_{\beta=1}^{q} \frac{1}{\lambda-a_{\beta}} \frac{\partial \mathcal{F}}{\partial a_{\beta}}+\oint_{\mathcal{C}_{\mathcal{D}}} \frac{d \xi}{2 \pi i} \frac{V^{\prime}(\xi)}{\lambda-\xi} W(\xi)=W(\lambda)^{2}+\hbar^{2} W(\lambda, \lambda) .
$$

We introduce the linear integral operator $\widehat{K}$,

$$
\widehat{K} f(\lambda) \equiv \oint_{\mathcal{C}_{\mathcal{D}}} \frac{d \xi}{2 \pi i} \frac{V^{\prime}(\xi)}{\lambda-\xi} f(\xi)=\left[V^{\prime}(\lambda) f(\lambda)\right]_{-}
$$


projects onto the negative powers ${ }^{1}$ of $\lambda$. Hereafter, $\mathcal{C}_{\mathcal{D}}$ is a contour encircling all singular points of $W(\xi)$, but not the point $\xi=p$. Using Eq. (6), one can express the second term in the r.h.s. of loop equation (13) through $W(\lambda)$, and Eq. (13) becomes then an equation for one-point resolvent (4).

Substituting genus expansion (8) in Eq. (13), one finds that $W_{h}(\lambda)$ for $h \geq 1$ satisfy the equation

$$
\sum_{\beta=1}^{q} \frac{1}{\lambda-a_{\beta}} \frac{\partial \mathcal{F}_{h}}{\partial a_{\beta}}+\left(\widehat{K}-2 W_{0}(\lambda)\right) W_{h}(\lambda)=\sum_{h^{\prime}=1}^{h-1} W_{h^{\prime}}(\lambda) W_{h-h^{\prime}}(\lambda)+\frac{\partial}{\partial V(\lambda)} W_{h-1}(\lambda),
$$

In Eq. (15), $W_{h}(\lambda)$ is expressed through only $W_{h_{i}}(\lambda)$ for which $h_{i}<h$. This fact allows developing the iterative procedure.

Note that the loop equation contains now explicit dependence on the points $a_{\beta}$. Although the term proportional to the derivative of $\mathcal{F}_{h}$ seems not to be universal, in what follows we find that these terms are important to keep the picture self-consistent.

We use the asymptotic condition (which follows from the definition of the matrix integral)

$$
\left.W_{h}(\lambda)\right|_{\lambda \rightarrow \infty}=\frac{t_{0}}{\lambda} \delta_{h, 0}+O\left(1 / \lambda^{2}\right),
$$

and manifestly solve (13) for genus zero. Then, one could iteratively find $W_{h}(\lambda)$ thus restoring the corresponding contributions in the free energy by integration, since

$$
W_{h}(\lambda)=-\frac{\partial}{\partial V(\lambda)} \mathcal{F}_{h}, \quad h \geq 1
$$

\subsection{Solution in genus zero}

In genus zero, loop equation (13) becomes

$$
\sum_{\beta=1}^{q} \frac{1}{\lambda-a_{\beta}} \frac{\partial \mathcal{F}_{0}}{\partial a_{\beta}}+\oint_{\mathcal{C}_{\mathcal{D}}} \frac{d \xi}{2 \pi i} \frac{V^{\prime}(\xi)}{\lambda-\xi} W_{0}(\xi)=\left[V^{\prime}(\lambda) W_{0}(\lambda)\right]_{-}+\frac{\bar{P}_{q-1}(\lambda)}{\prod_{\beta=1}^{q}\left(\lambda-a_{\beta}\right)}=\left(W_{0}(\lambda)\right)^{2},
$$

where the polynomial $\bar{P}_{q-1}(\lambda)$ is such that

$$
\sum_{\beta=1}^{q} \frac{1}{\lambda-a_{\beta}} \frac{\partial \mathcal{F}_{0}}{\partial a_{\beta}}=\frac{\bar{P}_{q-1}(\lambda)}{\prod_{\beta=1}^{q}\left(\lambda-a_{\beta}\right)} .
$$

\footnotetext{
${ }^{1}$ In order to prove it, one suffices to deform the integration contour to infinity to obtain

$$
\oint_{\mathcal{C}_{\mathcal{D}}} \frac{d \xi}{2 \pi i} \frac{V^{\prime}(\xi)}{\lambda-\xi} f(\xi)=V^{\prime}(\lambda) f(\lambda)-\left[V^{\prime}(\lambda) f(\lambda)\right]_{+}=\left[V^{\prime}(\lambda) f(\lambda)\right]_{-} .
$$
}


In what follows, for simplicity, we let $P_{r}(\lambda)$ denote any polynomial of $\lambda$ of order $r$ (which is indicated by the subscript). For instance, we can write $P_{r}(\lambda)+P_{u}(\lambda)=P_{w}(\lambda)$, where $w=\max (r, u)$.

In order to solve Eq. (18), note that

$$
\left[V^{\prime}(\lambda) W_{0}(\lambda)\right]_{-}=V^{\prime}(\lambda) W_{0}(\lambda)-\left[V^{\prime}(\lambda) W_{0}(\lambda)\right]_{+} .
$$

Due to (16), the last term in the r.h.s. is a polynomial of degree $m-1, m$ being the degree of $V^{\prime}(\lambda)$,

$$
P_{m-1}(\lambda)=-\left[V^{\prime}(\lambda) W_{0}(\lambda)\right]_{+}=-\oint_{\mathcal{C}_{\infty}} \frac{d \xi}{2 \pi i} \frac{V^{\prime}(\xi)}{\lambda-\xi} W_{0}(\xi)
$$

That is, we have

$$
W_{0}^{2}(\lambda)-V^{\prime}(\lambda) W_{0}(\lambda)=P_{m-1}(\lambda)+\frac{\bar{P}_{q-1}(\lambda)}{\prod_{\beta=1}^{q}\left(\lambda-a_{\beta}\right)}=\frac{P_{m+q-1}(\lambda)}{\prod_{\beta=1}^{q}\left(\lambda-a_{\beta}\right)} .
$$

Then, the solution to (18) is

$$
W_{0}(\lambda)=\frac{1}{2} V^{\prime}(\lambda)-\frac{1}{2} \frac{\sqrt{V^{\prime}(\lambda)^{2} \prod_{\beta=1}^{t}\left(\lambda-a_{\beta}\right)+4 P_{m+t-1}(\lambda)}}{\sqrt{\prod_{\beta=1}^{t}\left(\lambda-a_{\beta}\right)}},
$$

where the minus sign is chosen in order to fulfill asymptotics (16). We also assume that the general solution in the large- $N$ limit depends only on $t$ among $q$ hard wall points $a_{\beta}$. Then, from (19), we have that the corresponding pole disappear and does not enter expression (22). We therefore present the one-point resolvent in the form

$$
W_{0}(\lambda)=\frac{1}{2}\left(V^{\prime}(\lambda)-y(\lambda)\right),
$$

therefore introducing a new function $y(\lambda)$ satisfying the equation

$$
y(\lambda)^{2}=V^{\prime}(\lambda)^{2}+4 \frac{P_{m+t-1}(\lambda)}{\prod_{\beta=1}^{t}\left(\lambda-a_{\beta}\right)}
$$

and determined on a hyperelliptic Riemann curve. For the generic potential $V(\lambda)$ with $m \rightarrow \infty$, this curve may have an infinite genus, but we are going to consider solutions with only finite number $n$ of cuts. Endpoints of these cuts are $t$ points $a_{\beta}(t \geq q)$, which we enumerate by $\beta=1, \ldots, t$, and $s$ new endpoints $\mu_{\alpha}, \alpha=1, \ldots, s$. Apparently, $s+t=2 n$. We then present $y(\lambda)$ in the form

$$
y(\lambda) \equiv M(\lambda) \tilde{y}(\lambda), \quad \text { and } \quad \tilde{y}^{2}(\lambda) \equiv \frac{\prod_{\alpha=1}^{s}\left(\lambda-\mu_{\alpha}\right)}{\prod_{\beta=1}^{t}\left(\lambda-a_{\beta}\right)}
$$


with all $\mu_{\alpha}, a_{\beta}$ distinct. In what follows, we assume $M(\lambda)$ to be a polynomial of degree $m-\frac{s-t}{2}$, keeping in mind that both $s$ and $t$ are always finite and fixed, while $m \geq n$ can be chosen arbitrarily large. By convention, we set $\left.\tilde{y}(\lambda)\right|_{\lambda \rightarrow \infty} \sim \lambda^{(s-t) / 2}$, and due to (23),

$$
V^{\prime}(\lambda)=y(\lambda)+\frac{2 t_{0}}{\lambda}+O\left(1 / \lambda^{2}\right)
$$

at large $\lambda$.

In comparison with the standard 1MM situation, we may encounter an ambiguity. Indeed, as $\tilde{y}(\lambda)$ is proportional to $\lambda^{(s-t) / 2}$ as $\lambda \rightarrow \infty$, multiplying $W_{0}(\xi)$ by $\frac{\tilde{y}^{-1}(\xi)}{\xi-\lambda}$ and integrating at infinity may produce a nonzero result for $t>s$. For $M(\lambda)$, we then have

$$
M(\lambda)=\oint_{\mathcal{C}_{\infty}} \frac{d \xi}{2 \pi i} \frac{V^{\prime}(\xi)+P_{\frac{t-s}{2}}(\xi)-P_{\frac{t-s}{2}}(\lambda)}{(\xi-\lambda) \tilde{y}(\xi)} \equiv \prod_{i}^{m-\frac{s-t}{2}}\left(\lambda-b_{i}\right) .
$$

Here $P_{\frac{t-s}{2}}(\xi)$ is a polynomial and $b_{i}$ are zeros of $M(\lambda)$. In our consideration, we systematically avoid any explicit dependence on $b_{i}$. Instead, the relevant objects on which the free energy depends are moments $M_{i}^{(p)}$ of the general matrix model potential (see [34, 35, 17]) representing $(p-1)$ st derivatives of this polynomial at the branching points that are endpoints of intervals of eigenvalue distribution,

$$
M_{i}^{(p)}=\left.\frac{1}{(p-1) !}\left(\frac{\partial^{p-1}}{\partial \lambda^{p-1}} M(\lambda)\right)\right|_{\lambda=\mu_{i}}, \text { e.g., } M_{i}^{(1)}=M\left(\mu_{i}\right) .
$$

Among endpoints $\mu_{i}$ of these intervals there are hard wall points $a_{\beta}, \beta=1, \ldots, t(t \leq q)$ and a number of new "dynamical" endpoints $\mu_{\alpha}, \alpha=1, \ldots, s$ with $s+t=2 n$. Often (but not always) these points can be considered on the equal footing, as in formula (28), and we reserve the unified notation $\mu_{i}$ with the Latin subscript $i=1, \ldots, 2 n$ to indicate any branching point, whatever origin it has.

Directly from definition (28) follows the representation for the moments

$$
M_{i}^{(p)}=\oint_{\mathcal{C}_{\mathcal{D}}} \frac{d \xi}{2 \pi i} \frac{V^{\prime}(\xi)+P_{\frac{t-s}{2}}(\xi)-P_{\frac{t-s}{2}}(\lambda)}{\left(\xi-\mu_{i}\right)^{p} \tilde{y}(\xi)}, \quad p \geq 1 .
$$

Inserting solution (25), (27) into (23) and deforming the contour, we obtain the planar one-point resolvent with an $n$-cut structure,

$$
W_{0}(\lambda)=\frac{1}{2} \oint_{\mathcal{C}_{\mathcal{D}}} \frac{d \xi}{2 \pi i} \frac{V^{\prime}(\xi)+P_{\frac{t-s}{2}}(\xi)-P_{\frac{t-s}{2}}(\lambda)}{\lambda-\xi} \frac{\tilde{y}(\lambda)}{\tilde{y}(\xi)}, \quad \lambda \notin \mathcal{D} .
$$

The contour $\mathcal{C}_{\mathcal{D}}$ of integration here encircles the finite number $n$ of disjoint intervals

$$
\mathcal{D} \equiv \bigcup_{i=1}^{n}\left[\mu_{2 i-1}, \mu_{2 i}\right], \quad \mu_{1}<\mu_{2}<\ldots<\mu_{2 n}
$$


We now address the question how many free parameters do we have. In (30) we have $m$ times $t_{k}$ of the potential $V_{m}^{\prime}(\xi)$ (recall that the highest time is the unity), $\frac{t-s}{2}-1$ coefficients of the polynomial $P_{\frac{t-s}{2}}(\xi)$ (the constant term does not contribute) and $s$ new endpoints $\mu_{\alpha}$. However, we must take into account asymptotic conditions (16), which impose the restrictions

$$
\oint_{\mathcal{C}_{\mathcal{D}}} \frac{d \xi}{2 \pi i} \frac{\xi^{w} V^{\prime}(\xi)}{\tilde{y}(\xi)}=2 t_{0} \delta_{\frac{s-t}{2}, w}, \quad w=0,1, \ldots, \frac{s-t}{2},
$$

if $s \geq t$, or

$$
\oint_{\mathcal{C}_{\mathcal{D}}} \frac{d \xi}{2 \pi i} \frac{a_{t-s}}{\tilde{y}(\xi)}=2 t_{0}, \quad \text { where } \quad P_{\frac{t-s}{2}}(\lambda)=a_{\frac{t-s}{2}} \lambda^{\frac{t-s}{2}}+\ldots
$$

for $s<t$. If we do not count the hard wall parameters, in the first case, we have $m$ times of the potential, $s$ points $\mu_{\alpha}$, one zero time $t_{0}$ minus $\frac{s-t}{2}+1$ restrictions, that is $m+\frac{s+t}{2}=m+n$ parameters. In the second case, we have $m$ times of the potential, $s$ points $\mu_{\alpha}$, one zero time $t_{0}, \frac{t-s}{2}$ relevant coefficients of the polynomial $P_{\frac{t-s}{2}}(\lambda)$ and one restriction, which gives again $m+\frac{s+t}{2}=m+n$ free parameters. That means that, except the time $t_{0}$, we have an $n-1$-dimensional space of parameters. We can arbitrary choose coordinates on this space, but it turns out that there exists a distinguished set of $n-1$ independent variables that parameterize solutions to the loop equations [6, 3],

$$
S_{i}=\oint_{A_{i}} \frac{d \lambda}{4 \pi i} y(\lambda)=\oint_{A_{i}} \frac{d \lambda}{4 \pi i} M(\lambda) \tilde{y}(\lambda)
$$

where $A_{i}, i=1, \ldots, n-1$ is the basis of $A$-cycles on the reduced hyperelliptic Riemann surface of genus $h \equiv n-1$

$$
\widehat{y}^{2}=\prod_{\alpha=1}^{s}\left(\lambda-\mu_{\alpha}\right) \prod_{\beta=1}^{t}\left(\lambda-a_{\beta}\right) \equiv \prod_{i=1}^{2 n}\left(\lambda-\mu_{i}\right) .
$$

(We may conveniently choose the $A$-cycles to be the first $n-1$ cuts, see Fig.1.) Note that we do not distinguish the branching points $\mu_{i}$ in the above expression by their origin: both "dynamical" branching points $\mu_{\alpha}$ and hard walls $a_{\beta}$ enter $\widehat{y}(\lambda)$ on the equal footing. in contrast to the standard $1 \mathrm{MM}$ case, $\widehat{y}(\lambda) \neq \tilde{y}(\lambda)$. However, in what follows, $\widehat{y}(\lambda)$ enters some of the constructions of the paper.

Given the basis of $A$-cycles, we also choose the conjugated basis of $B$-cycles with the intersection form $A_{i} \circ B_{j}=\delta_{i j}$. Besides canonically conjugated $A$ - and $B$-cycles, we also use the linear combination of $B$-cycles: $\bar{B}_{i} \equiv B_{i}-B_{i+1}, \bar{B}_{n-1} \equiv B_{n-1}$. Therefore, $\bar{B}$-cycles encircle the nearest ends of two neighbor cuts, while all $B$-cycles goes from a given right end of the cut to the last, $n$th cut. For the sake of definiteness, we order all points $\mu_{i}$ in accordance with their index so that $\mu_{i}$ is to the right of $\mu_{j}$ if $i>j$.

\subsection{Matrix eigenvalue picture}

The variables $S_{i}$ find a "physical" interpretation in the semi-classical picture of matrix eigenvalues (Coulomb gas) in the limit where their number (and, therefore, size of the matrix) goes to infinity. 


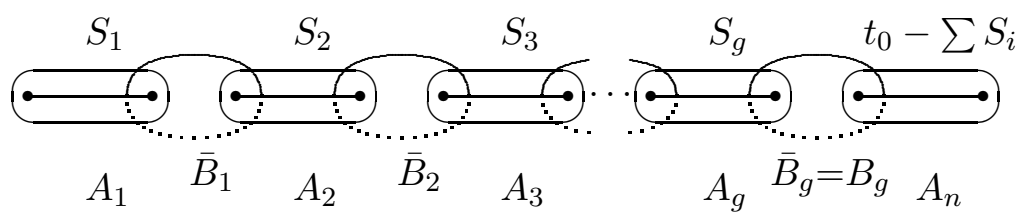

Figure 1: Structure of cuts and contours for the reduced Riemann surface.

Let us first introduce the averaged eigenvalue distribution

$$
\rho(\lambda) \equiv \frac{t_{0}}{N} \sum_{i}^{N}\left\langle\delta\left(\lambda-\lambda_{i}\right)\right\rangle=\frac{1}{2 \pi i} \lim _{\epsilon \rightarrow 0}(W(\lambda-i \epsilon)-W(\lambda+i \epsilon))
$$

with $\lambda_{i}$ being eigenvalues of the matrix $X$. In the planar limit, this quantity becomes

$$
\rho_{0}(\lambda)=\frac{1}{2 \pi i} \lim _{\epsilon \rightarrow 0}\left(W_{0}(\lambda-i \epsilon)-W_{0}(\lambda+i \epsilon)\right)=\frac{1}{2 \pi} \operatorname{Im} y(\lambda)
$$

and it satisfies the equation ${ }^{2}$

$$
\int_{\mathcal{D}} \frac{\rho_{0}(\lambda)}{x-\lambda} d \lambda=\frac{1}{2} V^{\prime}(x), \quad \forall p \in \mathcal{D} .
$$

This averaged eigenvalue distribution becomes the distribution of eigenvalues in the limit where their number goes to infinity. Then, matrix integral (9) becomes

$$
e^{\frac{1}{\hbar^{2}} \mathcal{F}}=\int_{E} \prod_{i} d x_{i} e^{-\frac{1}{\hbar^{2}}\left(\int V(\lambda) \varrho(\lambda)-\int \varrho(\lambda) \varrho\left(\lambda^{\prime}\right) \log \left|\lambda-\lambda^{\prime}\right| d \lambda d \lambda^{\prime}\right)} \equiv \int_{E} \prod_{i} d x_{i} e^{\frac{1}{\hbar^{2}} S_{\mathrm{eff}}}
$$

where we introduced the eigenvalue distribution

$$
\varrho(\lambda) \equiv \frac{t_{0}}{N} \sum_{i} \delta\left(\lambda-\lambda_{i}\right)
$$

Now, in the limit of large $N$, one can use the saddle point approximation to obtain the equation for $\varrho(\lambda)$. Since the variable $t_{0}$ plays the role of the (normalized) total number of eigenvalues,

$$
t_{0}=\frac{1}{4 \pi i} \oint_{\mathcal{C}_{\mathcal{D}}} y(\lambda) d \lambda=-\frac{1}{2} \operatorname{res}_{\infty}(y d \lambda)
$$

\footnotetext{
${ }^{2}$ Indeed, by definition

$$
\oint_{\infty} \frac{W_{0}(\lambda)}{x-\lambda} d \lambda=0
$$

Now, using (23) and definition (36) and pulling out the contour from infinity, one easily comes to this equation.
} 
and the support $\mathcal{D}$ of $\varrho$ consists of $n$ segments $\mathcal{D}_{i}$, we impose the constraint

$$
\int \varrho(\lambda) d \lambda=t_{0}
$$

and, following [6, 3], fix the occupation numbers of eigenvalues in each of the segments, $S_{i}$ (34), $i=1, \ldots, n-1$. We assume the occupation number for the last, $n$th cut to be $t_{0}-\sum_{i=1}^{n-1} S_{i} \equiv S_{n}$. ${ }^{3}$ (Obviously, no new parameters $S_{i}$ arise in the one-cut case.) We formally attain this by introducing the corresponding chemical potentials (Lagrange multipliers) $\Pi_{0}$ and $\Pi_{i}, i=1, \ldots, n-1$, in the variational problem for the free energy, which therefore becomes in the planar limit

$$
\begin{aligned}
S_{\mathrm{eff}}\left[\varrho ; S_{i}, t_{0}, t_{k}\right]= & -\int_{\mathcal{D}} V(\lambda) \varrho(\lambda) d \lambda+\iint_{\mathcal{D}} \varrho(\lambda) \log \left|\lambda-\lambda^{\prime}\right| \varrho\left(\lambda^{\prime}\right) d \lambda d \lambda^{\prime}+ \\
& -\Pi_{0}\left(\int_{\mathcal{D}} \varrho(\lambda) d \lambda-t_{0}\right)-\sum_{i=1}^{n-1} \Pi_{i}\left(\int_{D_{i}} \varrho(\lambda) d \lambda-S_{i}\right),
\end{aligned}
$$

while the saddle-point equation becomes

$$
2 \int \varrho(\lambda) \log |x-\lambda| d \lambda=V(x)+\Pi_{i}+\Pi_{0}, \quad \forall x \in \mathcal{D}_{i},
$$

and its derivative still coincides with (38).

Therefore, with generic values of the constants $\Pi_{i}, \varrho_{c}(\lambda)$ gives the general solution to (38) (or the planar limit of the loop equation): these constants describe the freedom arising when solving the loop equation. However, in the matrix model integral (where there are no any chemical potentials) one would further vary $\mathcal{F}_{0}$ w.r.t. $\Pi_{i}$ to find the "true" minimum of the eigenvalue configuration,

$$
\frac{\partial \mathcal{F}_{0}}{\partial S_{i}}=0, \quad \forall i
$$

This is a set of equation that fixes concrete values of $S_{i}$ and $\Pi_{i}$ in the matrix integral.

Let us now calculate the derivative of $\mathcal{F}_{0}$ (43) w.r.t. $S_{i}$. From (43), one has

$$
\left.\frac{\partial S_{\mathrm{eff}}}{\partial S_{i}}\right|_{\varrho=\rho_{0}}=-\int_{\mathcal{D}} d \lambda \frac{\partial \rho_{0}(\lambda)}{\partial S_{i}}\left(V(\lambda)-2 \int_{\mathcal{D}} d \lambda^{\prime} \log \left(\lambda-\lambda^{\prime}\right) \rho_{0}\left(\lambda^{\prime}\right)\right) .
$$

The expression in the brackets on the r.h.s. of (46) is almost a variation of (43) w.r.t. the eigenvalue density, which is

$$
\begin{gathered}
0=\left.\frac{\delta S_{\text {eff }}}{\delta \rho(\lambda)}\right|_{\varrho=\rho_{0}}=\quad V(\lambda)-2 \int_{\mathcal{D}} d \lambda^{\prime} \log \left(\lambda-\lambda^{\prime}\right) \rho_{0}\left(\lambda^{\prime}\right)+\Pi_{i}+\Pi_{0} \\
\quad \text { for } \lambda \in D_{i} \subset \mathcal{D} .
\end{gathered}
$$

\footnotetext{
${ }^{3}$ It is sometimes convenient to consider $S_{n}$ instead of $t_{0}$ as a canonical variable. However, in all instants we use $S_{n}$, we specially indicate it for not confusing $S_{n}$ with the "genuine" filling fraction variables $S_{i}, i=1, \ldots, n-1$.
} 
It is therefore a step function $h(\lambda)$, which is constant equal to $\zeta_{i} \equiv-\Pi_{0}-\Pi_{i}$ on each cut $A_{i}$. One then has

$$
\begin{aligned}
\frac{\partial \mathcal{F}_{0}}{\partial S_{i}}=\left.\frac{\partial S_{\mathrm{eff}}[\varrho]}{\partial S_{i}}\right|_{\varrho=\rho_{0}}=-\int_{\mathcal{D}} d \lambda \frac{\partial \rho_{0}(\lambda)}{\partial S_{i}} h(\lambda) & =-\frac{1}{4 \pi i} \sum_{j=1}^{n} \zeta_{j} \frac{\partial}{\partial S_{i}} \oint_{A_{j}} y(\lambda) d \lambda= \\
& =-\sum_{j=1}^{n} \zeta_{j} \frac{\partial S_{j}}{\partial S_{i}}=-\zeta_{i}+\zeta_{n}=\Pi_{i} .
\end{aligned}
$$

In particular,

$$
\frac{\partial \mathcal{F}_{0}}{\partial t_{0}}=\Pi_{0}
$$

In [4] it was proved that the difference of values of $\Pi_{i}$ on two neighbor cuts is equal to 4

$$
\zeta_{i+1}-\zeta_{i}=2 \int_{\mu_{2 i}}^{\mu_{2 i+1}} W_{0}(\lambda) d \lambda
$$

i.e.,

$$
\begin{aligned}
\Pi_{i} & =\left(\zeta_{i+1}-\zeta_{i}\right)+\left(\zeta_{i+2}-\zeta_{i+1}\right)+\ldots+\left(\zeta_{n-1}-\zeta_{n-2}\right)+\left(\zeta_{n}-\zeta_{n-1}\right) \\
& =\oint_{\bar{B}_{i} \cup \bar{B}_{i+1} \cup \ldots \cup \bar{B}_{g}} y(\lambda) d \lambda=\oint_{B_{i}} y(\lambda) d \lambda .
\end{aligned}
$$

The planar limit of the free energy can be obtained by substituting the saddle point solution $\varrho$ into (43):

$$
\mathcal{F}_{0}=S_{\text {eff }}\left[\varrho_{c}\right]=-\frac{1}{2} \int_{\mathcal{D}} V(\lambda) \varrho_{c}(\lambda) d \lambda+\frac{1}{2} \Pi_{0} t_{0}+\frac{1}{2} \sum_{i=1}^{n-1} \Pi_{i} S_{i} .
$$

In the paper, we choose the solution to the loop equation with fixed occupation numbers, (34). Note that fixing the chemical potentials (48)-(51) instead of (45), we obtain the solution with the interchanged $A$ - and $B$-cycles on the Riemann surface (24). However, $\mathcal{F}_{0}$ is not modular invariant. Under the change of homology basis, $\mathcal{F}_{0}$ transforms in accordance with the duality transformations, see 36]. The higher-genus corrections become also basis-dependent: choosing $S_{i}$ or $\Pi_{i}$ as independent variables, one obtains different expressions, say, for the genus-one free energy, see Sec. 4.3.

Note that the presence of hard walls has had no effect on consideration in this section because it only changes asymptotic behavior of the function $y(\lambda)$ near the corresponding branching points: while for "dynamical" branching points $\mu_{\alpha}$ the eigenvalue distribution undergoes the semicircular Wigner's law, it is known (see, e.g., [24]) that in the vicinity of hard wall we observe accumulation of

\footnotetext{
${ }^{4}$ The simplest way to prove it is to define function $h(\lambda)$ outside the cuts: $h(\lambda)=V(\lambda)-2 \int_{\mathcal{D}} d \lambda^{\prime} \log \left(\lambda-\lambda^{\prime}\right) \rho_{0}\left(\lambda^{\prime}\right)$ and note that $\left.h^{\prime}(\lambda)\right|_{\lambda \notin \mathcal{D}}=2 W_{0}(\lambda)$.
} 
eigenvalues (zeros of the corresponding orthogonal polynomials) with the reciprocal square-root law. This perfectly matches with our answer (25) for $y(\lambda)$.

In the next subsection, we demonstrate that the planar loop equation solution with fixed occupation numbers corresponds to a Seiberg-Witten-Whitham-Krichever system, just as in the case of ordinary $1 \mathrm{MM}[20$.

\subsection{Free energy as the prepotential of SWWK system}

We now turn to studying geometrical properties hidden in the matrix-model solutions. Namely, we associate a Seiberg-Witten-Whitham-Krichever (SWWK) system with the planar limit of the matrixmodel free energy.

Matrix integral as a Seiberg-Witten system. The family of Riemann surfaces is now the family of $g=n-1$ reduced Riemann surfaces described by (24) or (25). This means that these Riemann surfaces contain no information about the additional polynomial $M(\lambda)$, which is present, however, through (25) in the differential $d S$. The role of SW differential is played by

$$
d S=y(\lambda) d \lambda .
$$

We now consider its variation w.r.t. $S_{i}$. Variations over moduli of the surface do not change the genus of the reduced Riemann surface as well as the highest degree of the additional polynomial $M_{m-n}(\lambda)$. We also consider both the times of the potential $V_{m}^{\prime}(\lambda)$ and the hard wall parameters $a_{\beta}$ to be independent on the parameters $S_{i}$ and $t_{0}$, that is, we assume $\delta V^{\prime} / \delta S_{i} \equiv 0$ and consider the general variation $\delta$, which varies the potential and the branching points $\mu_{\alpha}$ (but not the hard wall parameters $a_{\beta}$ and the special variation $\delta_{S}$ that leaves invariant the potential and the hard wall parameters and change only the moduli parameters $S_{i}$.

Using (24), (25), one obtains for the general variation $\delta d S:{ }^{5}$

$$
\begin{aligned}
\delta d S & =\delta\left(M_{m-\frac{s-t}{2}}(\lambda) \tilde{y}(\lambda)\right) d \lambda= \\
& =\frac{\prod_{\alpha=1}^{s}\left(\lambda-\mu_{\alpha}\right) \delta M_{m-\frac{s-t}{2}}(\lambda)+\frac{1}{2} M_{m-\frac{s-t}{2}}(\lambda) \delta \prod_{\alpha=1}^{s}\left(\lambda-\mu_{\alpha}\right)}{\widehat{y}(\lambda)} d \lambda
\end{aligned}
$$

where the polynomial expression in the numerator is of maximum degree $m+\frac{s+t}{2}-1=m+n-1$ (since the highest term of $M_{m-\frac{s-t}{2}}$ is fixed) and we have in the denominator the hyperelliptic coordinate (35). On the other hand, under $\delta_{S}$ which does not alter the potential, we obtain from (24), (25) that

$$
\delta_{S} d S=-\frac{1}{2} \frac{\delta_{S} 4 P_{m+t-1}(\lambda)}{M_{m-\frac{s-t}{2}}(\lambda) \tilde{y}(\lambda) \prod_{\beta=1}^{t}\left(\lambda-a_{\beta}\right)} d \lambda
$$

\footnotetext{
${ }^{5}$ Note that the variation $\delta$ differs nevertheless from loop insertion (17) because the former does not change, by definition, the degree of the polynomial $M(\lambda)$.
} 


$$
=-2 \frac{\delta_{S} P_{m+t-1}(\lambda)}{M_{m-\frac{s-t}{2}}(\lambda) \widehat{y}(\lambda)} d \lambda .
$$

Because this variation is just a particular case of (154), we obtain that zeros of $M_{m-\frac{s-t}{2}}(\lambda)$ in the denominator of (55) must be exactly cancelled by zeros of the polynomial $\delta_{S} P_{m+t-1}(\lambda)$ in the numerator, so the maximum degree of the polynomial in the numerator is $\frac{s+t}{2}-2=n-2$ (because, again, the highest-order term of $P_{m+t-1}(\lambda)$ is fixed by asymptotic condition (26) and is not altered by variations $\left.\delta_{S}\right)$. We then come to the crucial observation that the variation $\delta_{S} d S$ is holomorphic on curve (35), as it should be for the SW differential.

Moreover, considering specific variation w.r.t. $S_{i}$ with condition (34) taken into account, we find that

$$
\delta_{i, j}=\delta_{S_{i}} S_{j}=\delta_{S_{i}} \oint_{A_{j}} \frac{d \lambda}{4 \pi i} y(\lambda)=\oint_{A_{j}} \frac{d \lambda}{4 \pi i} \delta_{S_{i}} y(\lambda)
$$

and hence

$$
\frac{\partial d S}{\partial S_{i}}=\frac{H_{i}(\lambda) d \lambda}{\widehat{y}(\lambda)}=d \omega_{i}
$$

with $d \omega_{i}$ being the canonically normalized $\left(\frac{1}{4 \pi i} \oint_{A_{i}} d \omega_{i}=\delta_{i, j}\right)$ holomorphic 1-differentials on the reduced Riemann surface $\widehat{y}(\lambda)$. Here $H_{i}(\lambda)$ are polynomials of degrees $n-2$ (the degree is exact because each such polynomial has exactly one zero on each cycle $A_{j}$ for $j \neq i$ ).

Rewriting now (51) as $\frac{\partial \mathcal{F}_{0}}{\partial S_{i}}=\oint_{B_{i}} d S$, together with (56), we reconstruct the SW system [37] whose prepotential is the planar limit $\mathcal{F}_{0}$ of the $1 \mathrm{MM}$ free energy. From this formula and (56) we obtain the celebrated expression relating $\mathcal{F}_{0}$ and the period matrix $\tau_{i, j}$ of the Riemann surface:

$$
\frac{\partial^{2} \mathcal{F}_{0}}{\partial S_{i} \partial S_{j}}=\oint_{B_{i}} d \omega_{j} \equiv \tau_{i, j}
$$

Matrix integral as a Whitham-Krichever system. We now show that $\mathcal{F}_{0}$ enjoys additional geometrical structures arising when differentiating w.r.t. times $t_{k}$ and $t_{0}$ thus accommodating the whole SWWK system.

We consider variations of the potential, i.e., variations w.r.t. (Whitham) times $t_{k}$. We then obtain instead of (55)

$$
\delta d S=-\frac{1}{2} \frac{\delta\left(\left(V_{m}^{\prime}\right)^{2}(\lambda)-\frac{P_{m+t-1}(\lambda)}{\prod_{\beta=1}^{t}\left(\lambda-a_{\beta}\right)}\right)}{M_{m-\frac{s-t}{2}}(\lambda) \tilde{y}(\lambda)} d \lambda
$$

while (54) still holds. Repeating the arguments of the previous paragraph, we conclude that the zeroes of $M_{m-\frac{s-t}{2}}(\lambda)$ cancel from the denominator and, therefore, the variation may have pole only at $\lambda=\infty$, or $\eta=0$, i.e., at the puncture. In order to find this pole, we use (54), which implies 
that $d S=M_{m-\frac{s-t}{2}}(\lambda) \tilde{y}(\lambda) d \lambda \rightarrow\left(V_{m}^{\prime}(\lambda)+O\left(\frac{1}{\lambda}\right)\right) d \lambda$ and, therefore, the variation of $d S$ at large $\lambda$ is completely determined by the variation of $V_{m}^{\prime}(\lambda)$. Parameterizing $V(\lambda)=\sum_{k=1}^{m+1} t_{k} \lambda^{k}$, we obtain that

$$
\frac{\partial d S}{\partial S_{i}}=d \omega_{i}, \quad \frac{\partial d S}{\partial t_{k}}=2 d \Omega_{k}
$$

with the behavior at infinity

$$
d \Omega_{k}=k\left(\xi^{-k-1}+O(1)\right) d \xi, \text { for } \xi \rightarrow 0, k>0 .
$$

This holds up to a linear combination of holomorphic differentials. The normalization of $d \Omega_{k}$ is fixed by the condition

$$
\frac{\partial S_{i}}{\partial t_{k}}=\frac{1}{2 \pi i} \oint_{A_{i}} d \Omega_{k}=0 \forall i, k, \quad \text { or } \quad \frac{\partial S_{i}}{\partial V(\lambda)}=0 \quad i=1, \ldots, n-1 .
$$

The derivatives of $d S$ w.r.t. the times are

$$
2 d \Omega_{k} \equiv \frac{\partial d S}{\partial t_{k}}=\frac{H_{n+k-1}(\lambda) d \lambda}{\widehat{y}(\lambda)}
$$

and normalization conditions (61) together with the asymptotic expansion

$$
\begin{aligned}
\left.2 d \Omega_{k}(\lambda)\right|_{\lambda \rightarrow \infty} & =k \lambda^{k-1} d \lambda+O\left(\lambda^{-2}\right) d \lambda= \\
& =k \lambda^{k-1} d \lambda+\sum_{m=1}^{\infty} c_{k m} \lambda^{-1-m} d \lambda .
\end{aligned}
$$

fixes uniquely the coefficients of the corresponding polynomials $H_{n+k-1}$ of degrees $n+k-1$.

Equations (59) together with normalization conditions (61) determine the SWWK system [7] (see also [40, 8]) whose prepotential is $\mathcal{F}_{0}$. For this, we apply the formula similar to (46) allowing now variations of the potential $V(\lambda)$. We then obtain (see (46)-(48))

$$
\begin{aligned}
\frac{\partial \mathcal{F}_{0}}{\partial t_{k}} & =-\frac{1}{4 \pi i} \oint_{\mathcal{D}} d \lambda \frac{\partial y(\lambda)}{\partial t_{k}} \cdot h(\lambda)-\frac{1}{4 \pi i} \oint_{\mathcal{D}} d \lambda y(\lambda) \lambda^{k}=-\sum_{i=1}^{n-1} \frac{\partial S_{i}}{\partial t_{k}}\left(\zeta_{i}-\zeta_{n}\right)+\frac{1}{2} \operatorname{res}_{\lambda=\infty}\left(\lambda^{k} d S\right) \\
& =\frac{1}{2} \operatorname{res}_{\lambda=\infty}\left(\lambda^{k} d S\right) \equiv \frac{1}{2} v_{k}, \quad k=1, \ldots, m,
\end{aligned}
$$

which is again the standard formula of the SWWK theory.

We now consider the variation of $d S$ w.r.t. the hard wall parameters $a_{\beta}$. Note first that because $S_{i}$ and $t_{0}$ are now independent variables, together with $t_{k}$ and $a_{\beta}$, we must demand

$$
\frac{\partial S_{i}}{\partial a_{\beta}}=\frac{1}{2 \pi i} \oint_{A_{i}} \frac{\partial y(\lambda)}{\partial a_{\beta}} d \lambda=0 \forall i, \beta, \quad \text { and } \quad \frac{\partial V^{\prime}(\lambda)}{\partial a_{\beta}}=0 .
$$


Performing the variation of (24) w.r.t. $a_{\beta}$, we obtain

$$
y(\lambda) \delta_{a_{\beta}} y(\lambda)=2 \frac{\delta_{a_{\beta}} P_{m+t-1}(\lambda)}{\sqrt{\prod_{\gamma=1}^{t}\left(\lambda-a_{\gamma}\right)}}+\frac{P_{m+t-1}(\lambda)}{\left(\lambda-a_{\beta}\right) \sqrt{\prod_{\gamma=1}^{t}\left(\lambda-a_{\gamma}\right)}}=\frac{\bar{P}_{m+t-1}(\lambda)}{\left(\lambda-a_{\beta}\right) \sqrt{\prod_{\gamma=1}^{t}\left(\lambda-a_{\gamma}\right)}},
$$

so, again, using considerations on cancelation of zeros of the polynomial $M(\lambda)$, we have

$$
\frac{\partial y(\lambda)}{\partial a_{\beta}}=\frac{\tilde{P}_{n-1}(\lambda)}{\left(\lambda-a_{\beta}\right) \widehat{y}(\lambda)} \equiv d \Omega_{a_{\beta}}(\lambda)
$$

where $d \Omega_{a_{\beta}}(\lambda)$ is a (noncanonical) meromorphic 1-form with the only second-order pole at $\lambda=a_{\beta}$ and with all its $A$-cycle integrals vanishing due to condition (65). To find the quadratic residue of this form at $\lambda=a_{\beta}$, we need the most singular contribution when performing the variation of $y(\lambda)=M(\lambda) \tilde{y}(\lambda)$ w.r.t. $a_{\beta}$; this contribution comes from the partial derivative in $a_{\beta}$, and we have

$$
d \Omega_{a_{\beta}}(\lambda)=\frac{1}{2} M\left(a_{\beta}\right) \frac{\sqrt{\prod_{\alpha=1}^{s}\left(a_{\beta}-\mu_{\alpha}\right)}}{\sqrt{\prod_{\substack{\gamma=1 \\ \gamma \neq \beta}}^{t}\left(a_{\beta}-a_{\gamma}\right)}} \frac{d \lambda}{\left(\lambda-a_{\beta}\right)^{3 / 2}}+O\left(\frac{1}{\sqrt{\lambda-a_{\beta}}}\right) d \lambda ; \quad \oint_{A_{i}} d \Omega_{a_{\beta}}(\lambda)=0 \forall i
$$

On $t_{0}$-dependence of the prepotential. The last property of $\mathcal{F}_{0}$ to be verified is the behavior w.r.t. $t_{0}$. From the SWWK theory, we expect that

$$
\frac{\partial \mathcal{F}_{0}}{\partial t_{0}}=\int_{\infty_{-}}^{\infty_{+}} d S
$$

and $\frac{\partial d S}{\partial t_{0}}=d \Omega_{0}$ where $d \Omega_{0}$ is the Abelian differential of the third kind with two simple poles at two infinities:

$$
\operatorname{res}_{\infty} d \Omega_{0}=-\mathrm{res}_{\infty_{-}} d \Omega_{0}=-1 .
$$

The integral in (69) is however divergent and needs regularization.

We know the derivative $\partial \mathcal{F}_{0} / \partial t_{0}$, (49), which is equal to the integral $\oint_{\mathcal{D}} \log |\lambda-q| d S-V(q)$ with the reference point $q$ to be chosen on the last, $n$ th, cut, while the expression itself does not depend on the actual local position of the reference point. We choose it to be $\mu_{2 n}$ - the rightmost point of the cut. We can then invert the contour integration over the support $\mathcal{D}$ to the integral along the contour that runs first along the upper side of the logarithmic cut from $\mu_{2 n}$ to a regularization point $\Lambda$, then over the circle $C_{\Lambda}$ of large radius $|\Lambda|$ and then back over the lower side of the logarithmic cut in the complex plane. In order to close the contour on the hyperelliptic Riemann surface under consideration, we must add the integration over the corresponding contour on the second sheet of the surface as shown in Fig. 2] we let $C_{L}$ denote the completed integration contour, and it is easy to see that such an additional integration just double the value of the integral. 


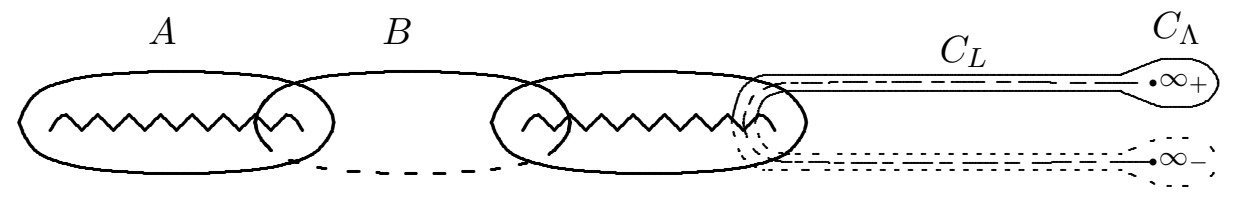

Figure 2: Cuts in the $\lambda$-, or "eigenvalue," plane for the planar limit of 1MM. The eigenvalues are supposed to be located "on" the cuts. We add the logarithmic cut between two copies of the infinity on two sheets of the hyperelliptic Riemann surface in order to calculate the derivative w.r.t. the variable $t_{0}$ and let $C_{L}$ denote the corresponding integration contour.

All the singularities appearing at the upper integration limit (i.e., at the point $\Lambda$ ) are exactly cancelled by the contribution coming when integrating the expression $d S \log \left(\lambda-\mu_{2 n}\right)$ along the circle $C_{\Lambda}$; in fact, the latter can be easily done, the result is $-2 \pi i\left(S(\Lambda)-\left(S\left(\mu_{2 n}\right)\right)_{+}\right)$, where the function $S(\lambda)$ is the (formal) primitive of $d S$ (which includes the logarithmic term), and we project it to the strictly polynomial part. Using the large- $\lambda$ asymptotic expansion of the differential $d S$,

$$
\left.d S(\lambda)\right|_{\lambda \rightarrow \infty}=V^{\prime}(\lambda) d \lambda+\frac{t_{0}}{\lambda} d \lambda+O\left(\lambda^{-2}\right) d \lambda,
$$

we obtain that $\left(S\left(\mu_{2 n}\right)\right)_{+}$just cancels the term $V\left(\mu_{2 n}\right)$, and we eventually find that

$$
\begin{aligned}
\frac{\partial F}{\partial t_{0}} & =\frac{1}{2}\left(\oint_{C_{L}} \log \left(\lambda-\mu_{2 n}\right) d S-2 V\left(\mu_{2 n}\right)\right)= \\
& =2 \pi i\left(\int_{\mu_{2 n}}^{\Lambda} d S-S(\Lambda)\right),
\end{aligned}
$$

where $C_{L}$ is the contour in Fig. 2, which by convention encircles the logarithmic cut between two infinities on two sheets of the Riemann surface and passes through the last, $n$ th, cut. We have therefore proved that the planar limit $\mathcal{F}_{0}$ of the $1 \mathrm{MM}$ free energy is the SWWK prepotential, or semiclassical tau-function.

We now introduce the (complete) set of canonical variables $\left\{S_{i}, i=1, \ldots, n-1 ; t_{0} ; t_{k}, k=\right.$ $1, \ldots, m\}$, which we uniformly denote $\left\{t_{I}\right\}$ (in what follows, Latin capitals indicate any quantity from this set). From (56), (70), and (62), we then obtain the general relation

$$
\frac{\partial d S}{\partial t_{I}} \equiv d \Omega_{I}=\frac{H_{I}(\lambda) d \lambda}{\widehat{y}(\lambda)}
$$

where $H_{I}(\lambda)$ are polynomials. 
Asymptotic formulas (70) and (63) imply that derivatives of all the quantities $d \Omega_{I}$ w.r.t. any parameter are regular at infinity and may have singularities only at the branching points $\mu_{i}$ of reduced Riemann surface (35).

Note again that these derivatives are purely geometrical and does not depend, in our setting, on the nature of the branching points: whether they come as hard walls or as "dynamical" branching points.

\section{Derivatives of the free energy and geometry}

\subsection{Second derivatives of the free energy}

Bergmann bidifferential and 2-point resolvent. Calculating the one-point resolvent in the preceding section required the knowledge of the first derivatives of the matrix model free energy. We now turn to the two-point resolvents involving second derivatives. Here, instead of differentials with some prescribed properties of holomorphicity, the main object we need is a bidifferential on a Riemann surface $\Sigma_{g}$ : the Bergmann kernel (canonically normalized bidifferential in Fay's terminology) which is the double derivative of the logarithm of the Prime form $E(P, Q)$ such that it is symmetrical in its arguments $P, Q \in \Sigma_{g}$ and has the only singularity at the coinciding arguments with the behavior (see 41])

$$
B(P, Q)=\left(\frac{1}{(\xi(P)-\xi(Q))^{2}}+O(1)\right) d \xi(P) d \xi(Q),
$$

in some local coordinate $\xi(P)$. As it stands, we can add to (74) any bilinear combination of Abelian 1differentials $d \omega_{i}$; we fix the normalization claiming vanishing all the integrals over $A$-cycles of $B(P, Q)$ :

$$
\oint_{A_{i}} B(P, Q)=0, \text { for } i=1, \ldots, g
$$

and, due to the symmetricity property, the integral may be taken over any of the variables $P$ or $Q$.

We now show that the Bergmann kernel generates the differentials $d \Omega_{k}$. Bipole differential (70) can be expressed through the Prime form as

$$
d \Omega_{0}=d \log \frac{E\left(P, \infty_{+}\right)}{E\left(P, \infty_{-}\right)}
$$

The primitive of differential (76) (which we need in what follows) then obviously develops the logarithmic cut between the points of two infinities on the Riemann surface.

Using (6), (64) and (59), we obtain

$$
W_{0}(\lambda, \mu) d \lambda d \mu=-\sum_{j=0}^{\infty} \frac{d \mu d \lambda}{\lambda^{j+1}} \frac{\partial}{\partial t_{j}} W_{0}(\mu)=-\frac{1}{2} \frac{\partial}{\partial V(\lambda)} V^{\prime}(\mu) d \lambda d \mu+\frac{1}{2} \sum_{j=0}^{\infty} \frac{d \lambda d \mu}{\lambda^{j+1}} \frac{\partial}{\partial t_{j}} y(\lambda)
$$




$$
=-\frac{1}{2} \frac{d \lambda d \mu}{(\lambda-\mu)^{2}}+\frac{1}{2} \sum_{j=0}^{\infty} \frac{d \lambda}{\lambda^{j+1}} d \Omega_{j}(\mu) .
$$

Because $d \Omega_{j}(\mu)=\left( \pm j \mu^{j-1}+O\left(1 / \mu^{2}\right)\right) d \mu$ for $\mu \rightarrow \infty_{ \pm}, \quad j>0$, we conclude that the infinite sum $\frac{1}{2} \sum_{j=0}^{\infty} \frac{d \lambda}{\lambda^{j+1}} d \Omega_{j}(\mu)$ develops the second-order pole $\frac{d \lambda d \mu}{2(\lambda-\mu)^{2}}$ in the case where $\lambda$ and $\mu$ are on the same (physical) sheet; this pole is then canceled by the first term, and the second-order pole $-\frac{d \lambda d \mu}{2(\lambda-\mu)^{2}}$ for $\lambda$ and $\mu$ being on different sheets. In the latter case, this pole is doubled by the first term, and together with the evident normalizing conditions that follow immediately from (77),

$$
\oint_{A_{i}} W_{0}(\lambda, \mu) d \mu=\oint_{A_{i}} W_{0}(\lambda, \mu) d \lambda=0
$$

we finally come to the formula for the 2-point resolvent,

$$
W_{0}(\lambda, \mu) d \lambda d \mu=\frac{\partial W_{0}(\lambda)}{\partial V(\mu)} d \lambda d \mu=-B\left(P, Q^{*}\right),
$$

and, correspondingly,

$$
\frac{\partial y(\lambda)}{\partial V(\mu)}=-\frac{1}{2}\left(B(P, Q)-B\left(P, Q^{*}\right)\right)
$$

where we have introduced the $*$-involution between two sheets of our hyperelliptic curve, so that $Q^{*}$ denotes the image of $Q$ under this involution. The only singularity of (79), for a fixed point $P$ on a physical sheet, is at the point $Q \rightarrow P^{*}$ on the unphysical sheet with $\mu(Q)=\lambda\left(P^{*}\right)=\lambda(P)$.

In what follows, we try as much as possible to express everything in terms of invariant quantities (the Bergmann bidifferentials and their primitives) on the reduced Riemann surface (35) and in terms of the WK differential $d S$. As we shall see, the $1 / N$-expansion can be constructed basically in terms of just these characteristics. However, we somewhere need the explicit expressions for $B(P, Q)$.

We need a technically simpler expression for the one-differential $d \mathcal{E}(\lambda, \mu)$, which is the primitive of the Bergmann kernel $B(\lambda, \mu)$ w.r.t. the argument $\mu .{ }^{6}$ Obviously, it is a single-valued differential of $\lambda$ with zero $A$-periods on the reduced Riemann surface and is multiple-valued function of $\mu$, which undergoes jumps equal to $d \omega_{i}(\lambda)$ when the variable $\mu$ passes along the cycle $B_{i}$ :

$$
\begin{aligned}
& d \mathcal{E}\left(P, Q+\oint_{B_{j}}\right)=2 \pi i d w_{j}(P)+d \mathcal{E}(P, Q), \\
& d \mathcal{E}\left(P, Q+\oint_{A_{j}}\right)=d \mathcal{E}(P, Q) .
\end{aligned}
$$

In Sec. 4, we use the explicit representation for the kernel $d \mathcal{E}(\lambda, \mu)$ in the hyperelliptic case:

$$
d \mathcal{E}(\lambda, \mu)=\frac{\widehat{y}(\mu) d \lambda}{(\lambda-\mu) \widehat{y}(\lambda)}-\sum_{i=1}^{n-1} \frac{H_{i}(\lambda) d \lambda}{\widehat{y}(\lambda)} \oint_{A_{i}} d \xi \frac{\widehat{y}(\mu)}{(\xi-\mu) \widehat{y}(\xi)} .
$$

\footnotetext{
${ }^{6}$ To be more precise, it is primitive of the antisymmetrized Bergmann kernel $\frac{1}{2}\left(B(\lambda, \mu)-B\left(\lambda^{*}, \mu\right)\right)$.
} 
We take $d \mathcal{E}(\lambda, \mu)$ to be antisymmetric w.r.t. the involution $\lambda \rightarrow \lambda^{*}$ between the physical and unphysical sheets, so it has the simple pole with the residue one at $\lambda=\mu$ and the simple pole with the residue minus one at $\lambda^{*}=\mu$ having vanishing $A$-periods in the variable $\lambda$. Then $d \mathcal{E}(\lambda, \mu)=\int_{\mu_{0}}^{\mu} B(\lambda, \xi)-$ $\int_{\mu_{0}}^{\mu^{*}} B(\lambda, \xi)$, with $\mu_{0}$ the reference point on which there is no dependence.

Note that formula (68) expressing the derivative of $y(\lambda)$ w.r.t. $a_{\beta}$ is proportional to $d \mathcal{E}\left(\lambda, a_{\beta}\right)$ and, moreover, can be conveniently written as the residue at the branching point $a_{\beta}$ :

$$
\frac{\partial y(\lambda) d \lambda}{\partial a_{\beta}} \equiv d \Omega_{a_{\beta}}(\lambda)=\operatorname{res}_{\mu=a_{\beta}}(B(\lambda, \mu) y(\mu)),
$$

whereas at a branching point $\mu_{i}(i=1, \ldots, 2 n)$, we have

$$
2 B\left(\lambda,\left[\mu_{i}\right]\right)=\lim _{\mu \rightarrow \mu_{i}} \frac{d \mathcal{E}(\lambda, \mu)}{\sqrt{\mu-\mu_{i}}} .
$$

In terms of $B\left(P,\left[\mu_{i}\right]\right)$, we have one of the Rauch variational identities [42]:

$$
\frac{\partial}{\partial \mu_{i}} B(P, Q)=\frac{1}{2} B\left(P,\left[\mu_{i}\right]\right) B\left(Q,\left[\mu_{i}\right]\right), \quad i=1, \ldots, 2 n .
$$

Mixed second derivatives. Normalizing conditions for $W_{h}(\lambda)$. Another set of relations follows from the general properties of the Bergmann kernel, and can be also derived directly from the formulas of Sec. 1. To this end, we apply the mixed derivatives $\partial / \partial V(\mu)$ and $\partial / \partial S_{i}$ to the planar limit of the free energy $\mathcal{F}_{0}$. On one hand, $\partial \mathcal{F}_{0} / \partial S_{i}=\oint_{B_{i}} d S$ and using that $d S(\lambda)=y(\lambda) d \lambda=\left(V^{\prime}(\lambda)-2 W_{0}(\lambda)\right) d \lambda$, $\frac{\partial V^{\prime}(\lambda)}{\partial V(\mu)}=-\frac{1}{(\lambda-\mu)^{2}}$ and formula (79), we have

$$
\oint_{B_{i}} \frac{\partial(d S(\lambda))}{\partial V(\mu)} d \mu=\oint_{B_{i}}\left(2 B(P, \mu)-\frac{1}{(\lambda-\mu)^{2}} d p d \mu\right)=\oint_{B_{i}} 2 B(P, \mu) .
$$

On the other hand, acting by derivatives in the opposite order, we first obtain $\partial \mathcal{F}_{0} / \partial V(\mu) d \mu=$ $W_{0}(\mu) d \mu=\left(V^{\prime}(\mu)-y(\mu)\right) d \mu$ and then $\partial\left(V^{\prime}(\mu)-y(\mu)\right) d \mu / \partial S_{i}=2 d \omega_{i}(\mu)$, or, in the coordinate-free notation, one of the classic identities (see, e.g., [41]):

$$
\frac{1}{2 \pi i} \oint_{B_{i}} B(P, Q)=d \omega_{i}(Q)
$$

This means that

$$
\frac{\partial d S(\mu)}{\partial S_{i}}=\left[\oint_{B_{i}} \frac{\partial d S(\lambda)}{\partial V(\mu)} d \lambda\right] d \mu=\oint_{B_{i}} \frac{\partial d S(\mu)}{\partial V(\lambda)} d \lambda
$$

where the both integrals are taken over the variable $\lambda$. Now, as $d S=y(\lambda) d \lambda$ is the generating function for the variables $\xi_{a} \equiv\left\{\left\{b_{i}\right\},\left\{\mu_{\alpha}\right\}, M_{\alpha}^{(i)}\right\}$, given by (25), (27), and (28) (recall that $M_{\alpha}^{(i)}$ are just $i$ th 
order derivatives of $M(\lambda)$ at $\lambda=\mu_{\alpha}$ ) giving their dependence on $S_{i}$ and $t_{k}$, one concludes that similar relation for the first derivatives holds for each of these variables. Indeed, multiplying (87) by $\frac{1}{y(\mu)}$ one then can bring $\mu$ successively to $\mu_{\alpha}$ 's, $\lambda_{i}$ 's and $\infty$ to pick up pole terms with different $\xi_{a}$ and prove that

$$
\frac{\partial \xi_{a}}{\partial S_{i}}=\oint_{B_{i}} \frac{\partial \xi_{a}}{\partial V(\lambda)} d \lambda
$$

which becomes true also for any function depending on a finite number of "local" variables $\xi_{a}$ in the case of arbitrary potential. For instance, it does not hold for $S_{j}$ for which $\partial S_{j} / \partial S_{i}=\delta_{i, j}$ whereas $\partial S_{j} / \partial V(\lambda) \equiv 0$. However, one can never express $S_{i}$ as a function of a finite number of "local" variables $\xi_{a}$.

Since, as we show in sec. 3, the free energy $\mathcal{F}_{h}$ at any order of $1 / N$-expansion depends only on a finite number of local variables, which are the branching points $\mu_{\alpha}$ and a finite number of moments $M_{\alpha}^{(i)}$, and $W_{h}(\lambda)$ is then expressed exclusively in terms of a finite number of derivatives $\frac{\partial \mu_{\alpha}}{\partial V(\lambda)}$ and $\frac{\partial M_{\alpha}^{(k)}}{\partial V(\lambda)}$, we have

$$
\frac{\partial \mathcal{F}_{h}}{\partial S_{i}}=\oint_{B_{i}} \frac{\partial \mathcal{F}_{h}}{\partial V(\lambda)} d \lambda=\oint_{B_{i}} W_{h}(\lambda) d \lambda \quad \forall h \text { and } i=1, \ldots, g,
$$

together with the condition

$$
\oint_{A_{i}} \frac{\partial \mathcal{F}_{h}}{\partial V(\lambda)} d \lambda \equiv \oint_{A_{i}} W_{h}(\lambda) d \lambda=0 \quad \forall h \geq 1 \text { and for } i=1, \ldots, g
$$

that follows directly from normalization condition (75).

In the same way, we can obtain the defining relation for the $t_{0}$-derivative

$$
\frac{\partial \mathcal{F}_{h}}{\partial t_{0}}=\int_{\infty_{-}}^{\infty_{+}} \frac{\partial \mathcal{F}_{h}}{\partial V(\xi)} d \xi
$$

which again holds for any $h$ and requires regularization only for $h=0$ since higher corrections in $h$ are regular at infinities.

\subsection{Residue formula and WDVV equations}

The Witten-Dijkgraaf-Verlinde-Verlinde (WDVV) equations [27, 28] is the systems of algebraic equations [29]

$$
\mathcal{F}_{I} \mathcal{F}_{J}^{-1} \mathcal{F}_{K}=\mathcal{F}_{K} \mathcal{F}_{J}^{-1} \mathcal{F}_{I}, \quad \forall I, J, K
$$

on the third derivatives

$$
\left\|\mathcal{F}_{I}\right\|_{J K}=\frac{\partial^{3} \mathcal{F}}{\partial t_{I} \partial t_{J} \partial t_{K}} \equiv \mathcal{F}_{I J K}
$$


of some function $\mathcal{F}\left(\left\{t_{I}\right\}\right)$. These equations can be often interpreted as associativity relations in some algebra for an underlying topological theory.

That the 1MM free energy satisfy the WDVV equations was proved in [20, where it was shown that the multicut solution of the 1MM satisfies the WDVV equations as a function of canonical variables identified with the periods and residues of the generating meromorphic one-form $d S$ [7]. The method to prove it consists of two steps. The first, most difficult, step is to find the residue formula for third derivatives (93) of the 1MM free energy. Then, using an associativity, one immediately proves that the free energy of multi-support solution satisfies the WDVV equations if we keep the number of independent variables to be equal to the number of "dynamical" branching points. That is, the presence of the hard-wall branching points results in the reducing the total dimension of the WDVV system.

Residue formula Because all the quantities $d \Omega_{I}(73)$ depend entirely on reduced hyperelliptic Riemann surface (35), their derivatives w.r.t. any parameter must be expressed through the derivatives w.r.t. the positions of the branching points $\mu_{i}$. We skip most of details referring the reader to [7, 20].

The formula for the third derivative $\partial^{3} \mathcal{F}_{0} /\left(\partial t_{I} \partial t_{J} \partial \mu_{\alpha}\right) \equiv \mathcal{F}_{I J \alpha}^{(0)}$ easily follows from (84) when $t_{I}$ and $t_{J}$ are times of the potential, and one should use the Riemann bilinear identities in the case of other canonical variables.

We eventually have

$$
\mathcal{F}_{I J \alpha}^{(0)}=\operatorname{res}_{\mu_{\alpha}}\left(d \Omega_{I} \partial_{\alpha} \Omega_{J}\right)=\frac{H_{I}\left(\mu_{\alpha}\right) H_{J}\left(\mu_{\alpha}\right)}{\prod_{j \neq \alpha}\left(\mu_{\alpha}-\mu_{j}\right)} .
$$

Completing the calculation of the third derivative needs just inverting the dependence on the branching points therefore finding $\partial \mu_{\alpha} / \partial t_{K}$. (Note that, obviously, $\partial a_{\beta} / \partial t_{K} \equiv 0$.) Differentiating expressions (24), (25) w.r.t. $t_{K}$, we obtain for (59)

$$
\begin{aligned}
& \frac{\partial d S(\lambda)}{\partial t_{K}}=\frac{H_{K}(\lambda) d \lambda}{\widehat{y}(\lambda)}= \\
& \quad=\frac{1}{2} M(\lambda) \sum_{\alpha=1}^{s} \frac{\tilde{y}(\lambda)}{\left(\lambda-\mu_{\alpha}\right)} \frac{\partial \mu_{\alpha}}{\partial t_{K}} d \lambda+\frac{\partial M(\lambda)}{\partial t_{K}} \tilde{y}(\lambda) d \lambda .
\end{aligned}
$$

The derivative of the polynomial $M(\lambda)$ is obviously polynomial and regular at $\lambda=\mu_{\alpha}$. Multiplying (95) by $\sqrt{\lambda-\mu_{\alpha}}$ and setting $\lambda=\mu_{\alpha}$, we immediately obtain

$$
\frac{\partial \mu_{\alpha}}{\partial t_{K}}=\frac{H_{K}\left(\mu_{\alpha}\right)}{M\left(\mu_{\alpha}\right) \prod_{\substack{\gamma=1 \\ \gamma \neq \alpha}}^{s}\left(\mu_{\alpha}-\mu_{\gamma}\right)},
$$

with only "dynamical" branching points in the product in the denominator. Combining this with (94), 
we come to the desired residue formula for the third derivative w.r.t. the canonical variables $t_{I}$ :

$$
\begin{aligned}
\frac{\partial^{3} \mathcal{F}_{0}}{\partial t_{I} \partial t_{J} \partial t_{K}} & =\sum_{\alpha=1}^{s} \frac{H_{I}\left(\mu_{\alpha}\right) H_{J}\left(\mu_{\alpha}\right) H_{K}\left(\mu_{\alpha}\right)}{M\left(\mu_{\alpha}\right) \prod_{\substack{\gamma=1 \\
\gamma \neq \alpha}}^{s}\left(\mu_{\alpha}-\mu_{\gamma}\right)^{2} \prod_{\beta=1}^{t}\left(\mu_{\alpha}-a_{\beta}\right)}= \\
& =\sum_{i=1}^{s} \operatorname{res}_{\mu_{\alpha}} \frac{d \Omega_{I} d \Omega_{J} d \Omega_{K}}{d \lambda d y}=\sum_{\alpha=1}^{2 n} \operatorname{res}_{\mu_{i}} \frac{d \Omega_{I} d \Omega_{J} d \Omega_{K}}{d \lambda d y}=\operatorname{res}_{d \lambda=0} \frac{d \Omega_{I} d \Omega_{J} d \Omega_{K}}{d \lambda d y} .
\end{aligned}
$$

We are able to pass to summation over all branching points in the last line because the residues at $\lambda=a_{\beta}$ just vanishes and in the very last expression we assume zeros of the differential $d \lambda$ on the reduced Riemann surface (35).

Proof of WDVV equations Given residue formula (97), the proof of WDVV equations (92) can be done by checking associativity of the algebra of differentials $d \Omega_{I}$ with multiplication modulo $\sqrt{\prod_{\alpha=1}^{s}\left(\lambda-\mu_{\alpha}\right)} d \lambda$. This algebra is reduced to the algebra of polynomials $H_{I}(\lambda)$ with multiplication modulo $\prod_{\alpha=1}^{s}\left(\lambda-\mu_{\alpha}\right)$, which is correctly defined and associative. The basis of the algebra of $H_{I}(\lambda)$ obviously has dimension $s$ and is given, e.g., by monomials of the degrees $0,1, \ldots, s-2, s-1$.

Another proof pertains to solving the system of linear equations [43, 44]. Defining

$$
\phi_{I}^{\alpha} \equiv \frac{H_{I}\left(\mu_{\alpha}\right)}{M^{1 / 3}\left(\mu_{\alpha}\right) \prod_{\substack{\gamma=1 \\ \gamma \neq \alpha}}^{s}\left(\mu_{\alpha}-\mu_{\gamma}\right)^{2 / 3} \prod_{\beta=1}^{t}\left(\mu_{\alpha}-a_{\beta}\right)^{1 / 3}},
$$

we reduce (97) to the form

$$
\mathcal{F}_{I J K}^{(0)}=\sum_{\alpha=1}^{s} \phi_{I}^{\alpha} \phi_{J}^{\alpha} \phi_{K}^{\alpha} .
$$

We also demand the determinant of $\phi_{I}^{\alpha}$ to be nonzero:

$$
\underset{I \alpha}{\operatorname{det}}\left\|\phi_{I}^{\alpha}\right\| \neq 0 .
$$

This nondegeneracy holds automatically in the case where we have no hard walls and choose the canonical variables to be $S_{i}, t_{0}$, and first $n$ times $t_{k}(k=1, \ldots, n)$ of the potential [21]. ${ }^{7}$ In the case

\footnotetext{
${ }^{7}$ From the definition [98, this condition stems, up to obviously nonvanishing Vandermonde determinant factors, to the nondegeneracy of the matrix $\sigma$ (145). Indeed, if $\operatorname{det} \sigma$ vanishes, then there exists a polynomial $P(\lambda)$ of degree less or equal $n-2$ such that

$$
\int_{\mu_{2 i-1}}^{\mu_{2 i}} \frac{P(\lambda)}{\widehat{y}(\lambda)} d \lambda=0 \quad \text { for } \quad i=1, \ldots, n-1 .
$$

This necessarily implies that $P(\lambda)$ has at least one zero at each of the intervals $\left(\mu_{2 i-1}, \mu_{2 i}\right)$; otherwise the combination under the integral sign is sign definite and the integral cannot vanish. The polynomial $P(\lambda)$ must then have at least $n-1$ zero and, having the degree not exceeding $n-2$, must therefore vanish.
} 
with hard walls, however, no such consideration works, and this determinant may vanish on subdomains of parameters of the problem, so we must consider condition (100) as an additional restriction, besides the matching condition claiming that the number of canonical variables must coincide with the number of "dynamical" branching points of the solution. Recall that we need at least three canonical variables in order to have a nontrivial WDVV system.

We now let $\mathcal{F}_{I}^{(0)}$ denote the matrix with the entry $J K$ equal to $\mathcal{F}_{I J K}^{(0)}$. The WDVV equations (92) follow now immediately from (99) and nondegeneracy condition (100).

\section{Higher genus contributions}

The solution $W_{1}(\lambda)$ to the loop equations in the multicut case was first found by Akemann [35] and the universality of critical behavior of the corresponding correlation functions was demonstrated in [5]. Akemann also managed to integrate the 1-point resolvent to obtain the free energy $\mathcal{F}_{1}$ in the two-cut case. The genus-one partition function in the generic multi-cut case was proposed in [45, 46], where it was observed that the Akemann formula coincides with the correlator of twist fields, computed by Al.Zamolodchikov [47. The function $F_{1}$ in the general multicut case was found by integrating $W_{1}(\lambda)$ in [48, 21].

\subsection{The iterative solution of the loop equation}

Inverting the integral operator. We now determine higher genus contributions. We do this iteratively by inverting the genus expanded loop equation (15). Our strategy will be to construct an integral operator $\widehat{d \mathcal{E}}$ inverse to the integral operator $\widehat{K}-2 W_{0}(\lambda)$.

Acting with this operator on the both sides of loop equation (15) we recursively obtain $W_{h}(\lambda)$ for all genera like all the multi-point resolvents of the same genus can be obtained from $W_{h}(\lambda)$ merely by

applying the loop insertion operator $\frac{\partial}{\partial V(\lambda)}$. Another ingredient of the construction will be the action of the loop insertion operator within our technique.

However, there is a subtlety: the operator $\widehat{K}-2 W_{0}(\lambda)$ has zero modes and is not invertible. Therefore, solution to the loop equation is determined up to an arbitrary combination of these zero modes. Moreover, in the case of hard walls, the kernel of $\widehat{d \mathcal{E}}$ does not coincide with the one of $\widehat{K}-2 W_{0}(\lambda)$ and must be discussed separately.

From representation (18) and (23), we have that the action of $\widehat{K}-2 W_{0}$ on $W_{h}(\lambda)$ is

$$
\begin{aligned}
\left(\widehat{K}-2 W_{0}(\lambda)\right) W_{h}(\lambda) & =\left[V^{\prime}(\lambda) W_{h}(\lambda)\right]_{-}-\left(V^{\prime}(\lambda)-y(\lambda)\right) W_{h}(\lambda) \\
& =\left[V^{\prime}(\lambda) W_{h}(\lambda)\right]_{-}-\left[\left(V^{\prime}(\lambda)-y(\lambda)\right) W_{h}(\lambda)\right]_{-} \\
& =\left[y(\lambda) W_{h}(\lambda)\right]_{-} .
\end{aligned}
$$


Because both $W_{h}(\lambda)$ and $y(\lambda)$ are odd functions under the involution $\lambda \rightarrow \lambda^{*}$ interchanging the physical and unphysical sheets, we conclude that the combination $y(\lambda) W_{h}(\lambda)$, being an even function under this involution, is a meromorphic function on the physical sheet, that is, it is a rational function having poles only at the branching points and at infinity.

It is now easy to see that the integral operator

$$
\widehat{d \mathcal{E}}(f) \equiv \oint_{\mathcal{C}_{\mathcal{D}}} \frac{d \mu}{2 \pi i} \frac{d \mathcal{E}(\lambda, \mu)}{d \lambda} \frac{1}{y(\mu)} \cdot f(\mu)
$$

with the kernel $d \mathcal{E}(\lambda, \mu)$ from (81) is an inverse for the operator $\widehat{K}-2 W_{0}(\lambda)$. Indeed, representing $f(\mu)$ in form (101), we have

$$
\begin{aligned}
& \oint_{\lambda>\mathcal{C}_{\mathcal{D}}} \frac{d \mu}{2 \pi i} \frac{d \mathcal{E}(\lambda, \mu)}{d \lambda} \frac{1}{y(\mu)}\left[y(\mu) W_{h}(\mu)\right]_{-}=\oint_{\lambda>\mathcal{C}_{\mathcal{D}}} \frac{d \mu}{2 \pi i} \frac{d \mathcal{E}(\lambda, \mu)}{d \lambda} \frac{1}{y(\mu)}\left(y(\mu) W_{h}(\mu)-P_{m-2}(\mu)\right) \\
& =\oint_{\lambda>\mathcal{C}_{\mathcal{D}}} \frac{d \mu}{2 \pi i} \frac{d \mathcal{E}(\lambda, \mu)}{d \lambda}\left(W_{h}(\mu)-\frac{P_{m-2}(\mu)}{y(\mu)}\right) \\
& =W_{h}(\lambda)+\oint_{\mathcal{C}_{\infty_{+}}} \frac{d \mu}{2 \pi i} \frac{d \mathcal{E}(\lambda, \mu)}{d \lambda} W_{h}(\mu)-\oint_{\lambda>\mathcal{C}_{\mathcal{D}}} \frac{d \mu}{2 \pi i} \frac{d \mathcal{E}(\lambda, \mu)}{d \lambda} \frac{P_{m-2}(\mu)}{y(\mu)}
\end{aligned}
$$

where $P_{m-2}(\mu)$ is a polynomial. Here, we indicated by $\lambda>\mathcal{C}_{\mathcal{D}}$ that the point $\lambda$ lies outside the integration contour in the physical sheet and, when pulling the contour of integration to $\infty_{+}$, only the residue at $\mu=\lambda$ contributes in the first integral and, by the normalization conditions, this residue is exactly $W_{h}(\lambda)$. The remaining integral at infinity vanishes because the integrand is regular as $\mu \rightarrow \infty$. Next, by virtue of explicit formula (81), we see that because the last term is a rational function on the physical sheet that is regular at the branching points, integrating it over $\mathcal{C}_{\mathcal{D}}$ obviously gives zero, so the whole action of $\widehat{d \mathcal{E}}$ on $\left(\widehat{K}-2 W_{0}\right) W_{h}(\lambda)$ just reconstructs $W_{h}(\lambda)$.

Note also that under the action on $\left[y(\mu) W_{h}(\mu)\right]_{-}$, due to the involution properties, we can replace the integration over $\mathcal{C}_{\mathcal{D}}$ just by evaluating residues at $\mu=\mu_{i}$ in formula (102) having

$$
\widehat{d \mathcal{E}}(f)(\lambda) d \lambda=\oint_{\mathcal{C}_{\left\{\mu_{i}\right\}}} \frac{d \mu}{2 \pi i} d \mathcal{E}(\lambda, \mu) \frac{1}{y(\mu)} \cdot f(\mu),
$$

where we let $\mathcal{C}_{\left\{\mu_{i}\right\}}$ denote the union of contours encircling all the branching points.

Because the operator $\widehat{d \mathcal{E}}$ obeys the property

$$
\oint_{A_{i}} \widehat{d \mathcal{E}}(f)(\lambda) d \lambda \equiv 0
$$

it also enjoys integrability conditions (90). Therefore, one has to solve the loop equations inverting $\widehat{K}-2 W_{0}$ exactly with $\widehat{d \mathcal{E}}$. 
Zero modes. The modified loop equation. Note that now, in contrast to the case without hard walls, the zero-mode content of the operators $\widehat{K}-2 W_{0}$ and $\widehat{d \mathcal{E}}$ is different.

It is clear from representation (101) that the zero modes of the operator $\widehat{K}-2 W_{0}$ when acting on the functions $W_{h}(\lambda)$ with asymptotic behavior (16) are spanned by $P_{\frac{s-t}{2}-2}(\lambda) / \tilde{y}(\lambda)$ and constitute the linear space of dimension $\frac{s-t}{2}-1$. In addition, all the simple-pole functions proportional to $\frac{1}{\lambda-a_{\beta}}$ are annihilated by the operator $\widehat{d \mathcal{E}}$. Altogether, it gives (in the case $s>t$ ) the total space of zero modes of dimension $\frac{s-t}{2}-1+t=n-1=g$, and the total number of zero modes under the action of the combination of the operators $\widehat{d \mathcal{E}} \circ\left(\widehat{K}-2 W_{0}\right)$ is therefore equal to the number $g$ of Abelian differentials, whereas the freedom to add these differentials is completely fixed by normalization condition (90).

A crucial observation is that when acting by the operator $\widehat{d \mathcal{E}}$ on the both sides of loop equation (15), the terms with the partial derivatives of $F_{h}$ w.r.t. $a_{\beta}$ vanish, the action of $\widehat{K}-2 W_{0}$ on $W_{h}(\lambda)$ is inverted, and the remained expression just becomes

$$
W_{h}(\lambda)=\widehat{d \mathcal{E}}\left(\sum_{h^{\prime}=1}^{h-1} W_{h^{\prime}}(\lambda) W_{h-h^{\prime}}(\lambda)+\frac{\partial}{\partial V(\lambda)} W_{h-1}(\lambda)\right)
$$

with $W_{h}(\lambda)$ now automatically satisfying normalization conditions (90).

It follows from the properties of the operator $\widehat{d \mathcal{E}}$ that, for all $s>0$ at $h>0$ and for $s \geq 3$ at $h=0$, the $s$-point resolvent $W_{h}\left(\lambda_{1}, \ldots, \lambda_{s}\right)$ possesses the gradation w.r.t. involution between variables of the physical and unphysical sheets:

$$
W_{h}\left(\lambda_{1}, \ldots, \lambda_{j}, \ldots \lambda_{s}\right)=-W_{h}\left(\lambda_{1}, \ldots, \lambda_{j}^{*}, \ldots \lambda_{s}\right)
$$

In particular, $W_{h}(\lambda, \lambda)$ as well as any product $W_{h}(\lambda) W_{h^{\prime}}(\lambda)$ in the r.h.s. of (106) are then rational functions of $\lambda$ for any $h, h^{\prime}>0$. Moreover, $W_{0}(\lambda, \lambda)=B\left(\lambda, \lambda^{*}\right)$ is again a rational function of $\lambda$ having poles of the second order at the point $\lambda=\mu_{i}$ of merging of the two sheets.

However, in order to have loop equation (15), not (106), we must demand additional conditions to be satisfied by the matrix-model free energy. In contrast to conditions (89) satisfied by any function of local variables, these conditions impose restrictions; indeed, it is required for the parts of loop equation (15) proportional to $\frac{1}{\lambda-a_{\beta}}$ to match. That is, the constructed loop means $W_{h}(\lambda)$ must satisfy

$$
\begin{aligned}
\frac{\partial \mathcal{F}_{h}}{\partial a_{\beta}} & =\oint_{\mathcal{C}_{a_{\beta}}} \frac{d \xi}{2 \pi i}\left(-\left[y(\xi) W_{h}(\xi)\right]_{-}+\sum_{h^{\prime}=1}^{h-1} W_{h^{\prime}}(\xi) W_{h-h^{\prime}}(\xi)+\frac{\partial}{\partial V(\xi)} W_{h-1}(\xi)\right) \\
& =\oint_{\mathcal{C}_{a_{\beta}}} \frac{d \xi}{2 \pi i}\left(-y(\xi) W_{h}(\xi)+\sum_{h^{\prime}=1}^{h-1} W_{h^{\prime}}(\xi) W_{h-h^{\prime}}(\xi)+\frac{\partial}{\partial V(\xi)} W_{h-1}(\xi)\right),
\end{aligned}
$$

because the residue at $\xi=a_{\beta}$ of the positive part $\left[y(\xi) W_{h}(\xi)\right]_{+}$is obviously zero. 
In two next paragraphs, we solve recurrently modified loop equation (106). We then integrate it to produce the free energy term $\mathcal{F}_{h}$ in the next section and, after obtaining the answer, must verify conditions (108). Note that the two normalization conditions, (89) and (108), leave no room for adding terms independent on $V(\xi)$ and dependent either on $S_{i}$ or on $a_{\beta}$ alone. And, as we show in the last section, in the special case of $\mathcal{F}_{1}$, condition (108) fixes the function of $a_{\beta}$ to be added to the free energy.

Diagrammatic technique. The above considerations provide a basis for the diagrammatic representation for resolvents, which almost literally repeats the diagrammatic technique for the $1 \mathrm{MM}$ without hard walls [22]. Let us represent the one-form $d \mathcal{E}(p, q)$ as the vector directed from $p$ to $q$, the three-point vertex as the dot in which we assume the integration over $q, \bullet \equiv \oint \frac{d q}{2 \pi i} \frac{1}{2 y(q)}$, and the Bergmann bidifferential $B(p, q)$ as a nonarrowed edge connecting points $p$ and $q$. The graphic representation for a solution of (106) then looks as follows. Representing the multiresolvent $W_{h^{\prime}}\left(p_{1}, \ldots, p_{s}\right)$ as the block with $s$ external legs and with the index $h^{\prime}$, we obtain 22 .

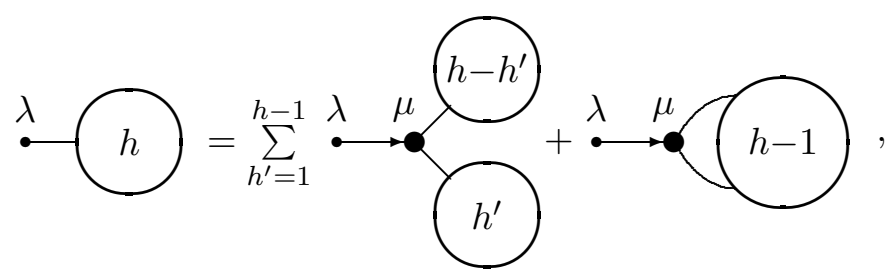

which provides the basis for the diagrammatic representation for $W_{h}\left(p_{1}, \ldots, p_{s}\right)$. It can be formulated as a set of the following diagrammatic rules [22].

The multiresolvent $W_{h}\left(p_{1}, \ldots, p_{s}\right)$ is presented as a finite sum of all possible connected graphs with $h$ loops and $s$ external legs such that

- only three-valent internal vertices are allowed (the total number of edges is then $2 s+3 h-3$, and we assume $s \geq 1$ for $h \geq 1$ and $s \geq 3$ for $h=0$ ); all the vertices are indexed by the corresponding coordinate variables $q$ on the Riemann surface $\widehat{y}(\lambda)$.

- We have propagators (edges) which either connect internal vertices (can start and terminate at the same vertex) or come as an external leg to an internal vertex.

- These propagators are of two sorts: there are arrowed propagators and nonarrowed propagators. As above, we set the 1-forms $d \mathcal{E}(p, q)$ into the correspondence to the arrowed propagators and the variables $p$ and $q$ correspond to the respective vertices at which this edge starts and terminates. We have the Bergmann bidifferential $B(p, q)$ corresponding to each nonarrowed edge that starts and terminates at the vertices labeled by the respective variables $p$ and $q$ and if it is the same vertex, then $B\left(p, p^{*}\right)$ corresponds to this propagator. 
- The arrangement of propagators is subject to restrictions. We have exactly $2 k+s-2$ arrowed edges; these edges must constitute the maximum rooted tree subgraph with all arrows directed from the root. Different choices of the tree subgraph for the same graph are considered different cases of the construction, and we must consider all possible cases. The root of the tree subgraph is always a selected (one and for all) external leg, say, $p_{1}$ (the choice is arbitrary due to the symmetry of $\left.W_{k}\left(p_{1}, \ldots, p_{s}\right)\right)$ and the maximality of the tree subgraph means that it contains no loops (including tadpoles; the arrowed propagators cannot therefore start and terminate at the same vertex), is connected, and for every internal vertex there is exactly one arrowed propagator that terminates at this vertex. We call this propagator the incoming propagator for this vertex. There can be either one, or two, or no outgoing arrowed propagators at an internal vertex of the graph.

- Each tree subgraph establishes the relation of partial ordering on the set of internal vertices of the graph; among two vertices one precedes to the other if there exist a naturally oriented path in the rooted arrowed tree subgraph that starts at the first vertex and terminates at the second vertex. At each internal vertex we place the integration $\oint_{\mathcal{C}_{\mathcal{D}}^{(q)}} \frac{d q}{2 \pi i} \frac{1}{2 y(q)}$ over the variable indexing this vertex; the integration goes along the contour that is about the domain $\mathcal{D}$ and the arrangement of the integration contours at different vertices is prescribed by the arrowed subtree: the closer is a vertex to the root, the more outer is the integration contour.

- All internal nonarrowed propagators are allowed to connect only vertices that are in the partial ordering relation to one another; it can be the same vertex. All external propagators except the one that is the root of the tree subgraph are nonarrowed, and we have the Bergmann bidifferentials $B\left(q, p_{k}\right) \quad(k=2, \ldots, s)$ corresponding to these edges.

- All the integration contours over the $A$-cycles in formula (81) for $d \mathcal{E}(\lambda, \mu)$ are assumed to be outside all the integration contours over the variables indexing the internal vertices whereas all the external points $p_{i}, i=1, \ldots, s$ are assumed to be outside all the integration contours $\mathcal{C}_{\mathcal{D}}^{(q)}$ (as well as outside all the contours of integration over the $A$-cycles).

We now demonstrate the consistency of this diagrammatic technique by calculating the action of loop insertion operator $\partial / \partial V(r)$ on its elements following [23].

We first calculate the action of $\partial / \partial V(r)$ on $B(P, Q)$ thus producing an analogue of formula (97). Using (84), we represent this action through the action of partial derivatives in $\mu_{\alpha}(\alpha=1, \ldots, s$; only the dynamical branching points participate on this stage) subsequently calculating the latter from relation (80). Let

$$
\left.y(x) d x\right|_{x \rightarrow \mu_{\alpha}}=y\left(\left[\mu_{\alpha}\right]\right) \sqrt{x-\mu_{\alpha}} d x+O\left(\sqrt{x-\mu_{\alpha}}\right)^{3} d x .
$$

Then, since

$$
\left.\frac{\partial y(p) d p}{\partial V(r)}\right|_{p \rightarrow \mu_{\alpha}} \simeq-\frac{1}{2} y\left(\left[\mu_{\alpha}\right]\right) \frac{d p}{\sqrt{p-\mu_{\alpha}}} \frac{\partial \mu_{\alpha}}{\partial V(r)}
$$


we have

$$
\frac{\partial \mu_{\alpha}}{\partial V(r)}=\frac{2 B\left(\left[\mu_{\alpha}\right], r\right)}{y\left(\left[\mu_{\alpha}\right]\right)}
$$

and, therefore

$$
\frac{\partial}{\partial V(r)} B(P, Q)=\sum_{\alpha=1}^{s} \frac{B\left(P,\left[\mu_{\alpha}\right]\right) B\left(Q,\left[\mu_{\alpha}\right]\right) B\left(r,\left[\mu_{\alpha}\right]\right)}{y\left(\left[\mu_{\alpha}\right]\right)} .
$$

This is apparently just formula (97) upon setting all variables $I, J, K$ to be the times of the potential with subsequent summation with $p^{-i-1}, q^{-j-1}$, and $r^{-k-1}$ for $p, q$, and $r$ being the variables on the physical sheet.

However, now we represent expression (111) in the different way. Instead of differentiating $y(\xi)$ in the denominator as we did in formula (97), we integrate one of the Bergmann kernels, which gives the 1-differential $d \mathcal{E}(Q, \xi)$. Note that the local variable in the vicinity of $\mu_{\alpha}$ is $\xi(x)=\sqrt{x-\mu_{\alpha}}$, this gives the additional factor $1 / 2$, so we have

$$
\frac{\partial}{\partial V(r)} B(P, Q)=\sum_{\alpha=1}^{s} \operatorname{res}_{\mu_{\alpha}} \frac{B(P, \xi(x)) d \mathcal{E}(Q, \xi(x)) B(r, \xi(x))}{2 y(\xi(x)) d \xi(x)},
$$

and we can again add residues at the hard wall points $a_{\beta}$ because they are just vanish here, so our final expression is

$$
\frac{\partial}{\partial V(r)} B(P, Q)=\sum_{i=1}^{2 n} \operatorname{res}_{\mu_{i}} \frac{B(P, \xi(x)) d \mathcal{E}(Q, \xi(x)) B(r, \xi(x))}{2 y(\xi(x)) d \xi(x)}
$$

The price for preserving $y(\xi(x)) d \xi(x)=d S$ in the denominator is that we have lost the explicit permutational symmetry for the three terms in the numerator, which was apparent in (97). We however pay this price for the possibility to develop the comprehensive diagrammatic technique. From this relation, it obviously follows that

$$
\frac{\partial}{\partial V(r)} d \mathcal{E}(Q, P)=\sum_{i=1}^{2 n} \operatorname{res}_{\mu_{i}} \frac{d \mathcal{E}(\xi(x), P) d \mathcal{E}(Q, \xi(x)) B(r, \xi(x))}{2 y(\xi(x)) d \xi(x)},
$$

and the last quantity to evaluate is

$$
\frac{\partial}{\partial V(r)} \frac{1}{2 y(p)}=-\frac{B(p, r)}{2 y^{2}(p)}
$$

Note that the point $P$ in (113) is outside the integration contour. Multiplying the both sides of (113) by $1 /(2 y(P))$, using (114), and pushing the integration contour through the point $P$, we observe that the contribution of the simple pole of $d \mathcal{E}(\xi(x), P)$ at the point $\xi(x)=P$ cancels exactly 
the variation of $1 /(2 y(P))$ ! We therefore attain the prescribed contour ordering and can graphically present the action of $\partial / \partial V(r)$ as

$$
\frac{\partial}{\partial V(r)} Q \longrightarrow P=Q \stackrel{r}{\longrightarrow} P, \quad \frac{\partial}{\partial V(r)} Q \longrightarrow P=Q \stackrel{r}{\perp} P \equiv Q \stackrel{r}{\llcorner} P .
$$

In the second case, we have the freedom to choose on which of edges to set the arrow. Recall, however, that as we have had a nonarrowed propagator stretched between the points $P$ and $Q$, these points were to be ordered before, as prescribed by the diagrammatic technique. That is, if " $P$ preceded $Q$ " we must choose the first variant and if " $Q$ preceded $P$ " we must choose the second variant of arrows arrangement.

\subsection{Inverting the loop insertion operator. Free energy}

The $H$-operator. We now introduce the operator that is inverse to loop insertion operator (7). Let $^{8}$

$$
H \cdot=\frac{1}{2} \operatorname{res}_{\infty_{+}} V(x) \cdot-\frac{1}{2} \operatorname{res}_{\infty_{-}} V(x) \cdot-t_{0} \int_{\infty_{-}}^{\infty_{+}} \cdot-\sum_{i=1}^{n-1} S_{i} \oint_{B_{i}} \cdot
$$

The arrangement of the integration contours is as in Fig. 2 We calculate the action of $H$ on the Bergmann bidifferential $B(x, q)$ using again the Riemann bilinear identities. We first note that as $B(x, q)=\partial_{x} d \mathcal{E}(q, x)$, we can evaluate residues at infinities by parts. Then, since $d \mathcal{E}(q, x)$ is regular at infinities, we substitute $2 y(x)+2 t_{0} / x$ for $V^{\prime}(x)$ as $x \rightarrow \infty_{+}$and $-2 y(x)+2 t_{0} / x$ for $V^{\prime}(x)$ as $x \rightarrow \infty_{-}$ thus obtaining

$$
\begin{aligned}
& -\operatorname{res}_{\infty_{+}}\left(y(x)+\frac{t_{0}}{x}\right) d \mathcal{E}(q, x) d x+\operatorname{res}_{\infty_{-}}\left(-y(x)+\frac{t_{0}}{x}\right) d \mathcal{E}(q, x) d x \\
& -\left.t_{0} d \mathcal{E}(q, x)\right|_{x=\infty_{-}} ^{x=\infty_{+}}-\sum_{i=1}^{n-1} S_{i} \oint_{B_{i}} B(q, x) .
\end{aligned}
$$

Whereas the cancelation of terms containing $t_{0}$ is obvious, it remains only to take the combination of residues at infinities involving $y(x)$. For this, we cut the surface along $A$ - and $B$-cycles taking into account the residue at $x=q$. The boundary integrals on two sides of the cut at $B_{i}$ then differ by $d \mathcal{E}(q, x)-d \mathcal{E}\left(q, x+\oint_{A_{i}}\right)=0$, while the integrals on the two sides of the cut at $A_{i}$ differ by $d \mathcal{E}(q, x)-d \mathcal{E}\left(q, x+\oint_{B_{i}}\right)=\oint_{B_{i}} B(q, x)$, and the boundary term therefore becomes

$$
\sum_{i=1}^{n-1} \oint_{A_{i}} y(x) d x \oint_{B_{i}} B(q, \xi)
$$

\footnotetext{
${ }^{8}$ This definition works well when acting on 1 -forms regular at infinities. Otherwise (say, in the case of $W_{0}(p)$ ), the integral in the third term must be regularized, e.g., by replacing it by the contour integral around the logarithmic cut stretched between two infinities as was done in Sec. 1.5
} 
which exactly cancels the last term in (117). Only the contribution from the pole at $x=q$ then survives, and this contribution is just $-y(q)$. We have therefore proved that

$$
H \cdot B(\cdot, q)=-y(q) d q
$$

Free energy. We now act by $H$ on $W_{k}(\cdot)$ subsequently evaluating the action of loop insertion operator (7) on the result. Note first that the only result of action of $\partial / \partial V(p)$ on the operator $H$ itself are derivatives $\partial V(x) / \partial V(p)=-1 /(p-x)$ (and recall that by definition $|p|>|x|$, i.e., instead of evaluating residues at infinities one should take residues at $x=p$ ), which gives

$$
\frac{\partial}{\partial V(p)}\left(H \cdot W_{h}(\cdot)\right)=W_{h}(p)+H \cdot W_{h}(\cdot, p)
$$

For the second term, due to the symmetry of $W_{h}(p, q)$, we may choose now the point $p$ as the root of all the tree subgraphs. Then, the operator $H$ always acts on $B(\cdot, \xi)$ where $\xi$ are integration variables of internal vertices. However, if this vertex is an innermost (i.e., it has no outgoing arrowed edges), then the 1-form $y(\xi) d \xi$ arising under the action of $H$ (118) cancels the corresponding 1-form in the denominator of the integrand, which becomes therefore regular at the branching point giving zero contribution. If this vertex has an outgoing arrowed edge, say $d \mathcal{E}(\xi, \rho)$ (it can be at most one outgoing arrowed edge as the third edge must be external), then, again, we can push the integration contour for $\xi$ through the one for $\rho$; the only contribution comes only from the pole at $\xi=\rho$. The value of the residue is however doubled, and we come to the following graphical representation for the action of the operator $H$ :

$$
Q \stackrel{H}{\rightarrow} P=-Q \longrightarrow P ; \quad Q \stackrel{H}{\rightarrow} P=0 .
$$

For $H_{q} \cdot W_{k}(q, p)=H_{q} \cdot \frac{\partial}{\partial V(q)} W_{k}(p)$, we obtain that for each arrowed edge on which the action of $\partial / \partial V(r)$ produces the new vertex, the inverse action of $H_{q}$. just give the factor -1 and for each nonarrowed edge on which the action of $\partial / \partial V(r)$ produces the new vertex, the inverse action of $H_{q}$. just gives zero. As the total number of arrowed edges is exactly $2 h-1$ for every graph contributing to the sum of diagrams with one external edge and containing $h$ loops, we obtain that

$$
H_{q} \cdot W_{h}(q, p)=-(2 h-1) W_{h}(p) .
$$

Therefore, combining with (119), we just obtain

$$
\frac{\partial}{\partial V(p)}\left(H_{q} \cdot W_{h}(q)\right)=-(2-2 h) \frac{\partial}{\partial V(p)} \mathcal{F}_{h},
$$


and, since all the dependence on filling fractions and $t_{0}$ is fixed by the claim that the answer depends only on $\mu_{i}$ and derivatives of $M(p)$ of finite orders at the branching points, we conclude that

$$
\mathcal{F}_{h}=\frac{1}{2 h-2} H \cdot W_{h}
$$

We have therefore expressed the free energy through the one-loop resolvent. Using this final answer, we can calculate all $\mathcal{F}_{h}$ except the contribution at $h=1$ (the torus approximation). In the case without hard walls, the latter was calculated by a direct integration in 48. (see the refined version in [21]), and we generalize this calculation to the hard wall case in Sec. 4. All other orders can be calculated consistently. For this, we need to introduce one new vertex $\odot$ in which we place the new integration term:

$$
\odot \sim \oint_{\mathcal{C}_{\left\{\mu_{i}\right\}}} \frac{d \xi}{2 \pi i} \frac{\int_{\mu_{i}}^{\xi} y(s) d s}{y(\xi)} .
$$

This is because, although the integral term $\int_{Q_{0}}^{\xi} y(s) d s$ is nonlocal, its constant part $\int_{Q_{0}}^{\mu_{i}}$, having the improper involution symmetry, drops out of the residue in the $1 \mathrm{MM}$ case, and we can integrate only in the neighborhood of each branching point $\mu_{i}$ separately. That is, we need only the local expansion of this integral as $\xi \rightarrow \mu_{i}$. Then, say, the genus two contribution is provided by the sum of three diagrams

$$
\left.2 \cdot \mathcal{F}_{2}=2 \oint \rightarrow \bigcirc+2 \oint i\right)+\bigcirc \rightarrow \leftrightarrow 0
$$

Note also that $\mathcal{F}_{0}$ written in form (52) obviously can be presented as

$$
\mathcal{F}_{0}=-\frac{1}{2} H_{\mathrm{reg}} \cdot W_{0}
$$

due to (48) and (49) and by virtue of relations (89) and (91). Recall that in the case of $\mathcal{F}_{0}$, the integral between $\infty_{-}$and $\infty_{+}$must be regularized as in Sec. 1.5.

Postponing the consideration of the case $\mathcal{F}_{1}$ to the next section, let us show here that $H \cdot W_{1}$ is constant. Indeed,

$$
H \cdot W_{1}=\sum_{i=1}^{2 n} \operatorname{res}_{\mu_{i}} \frac{\int_{\mu_{i}}^{\xi} y(s) d s}{y(\xi)} B\left(\xi, \xi^{*}\right) d \xi,
$$

and the first term has simple zero with residue $2 / 3$ or 2 (depending on which branching point - hard wall or dynamical - we have at $\left.\mu_{i}\right)$ to be compensated by the double pole of $B\left(\xi, \xi^{*}\right) \simeq \frac{1}{4\left(\xi-\mu_{\alpha}\right)^{2}}$, and the total answer is then just the constant equal to $(2 s / 3+2 t) / 4$. 
Scaling relation. We now explain the origin of relation (121). Indeed, recall that for any functional $\mathcal{F}$ of a finite number of "local" variables, which are in our case the branching points $\mu_{i}$ and the moments $M_{i}^{(k)}$, we have relations (89) and (91) that state that $\oint_{B_{i}} \frac{\partial \mathcal{F}_{h}}{\partial V(\xi)} d \xi=\partial \mathcal{F}_{h} / \partial S_{i}$ and that $\int_{\infty_{-}}^{\infty_{+}} \frac{\partial \mathcal{F}_{h}}{\partial V(\xi)} d \xi=\partial \mathcal{F}_{h} / \partial t_{0}$. Relation (121) is then equivalent to the formula

$$
\left[\sum_{k=1}^{\infty} t_{k} \frac{\partial}{\partial t_{k}}+t_{0} \frac{\partial}{\partial t_{0}}+\sum_{i=1}^{n-1} S_{i} \frac{\partial}{\partial S_{i}}+\hbar \frac{\partial}{\partial \hbar}\right] \mathcal{F}=0
$$

which is just a ("kinematic") property of every integral of form (11). Indeed, let us fix the total number of eigenvalues to be $N$ and the partial numbers of eigenvalues on intervals of distribution to be $N_{i}$. Then, $t_{0}=\hbar N, S_{i}=\hbar N_{i}$, and we have the exponential term $\frac{1}{\hbar} \sum_{k} t_{k} \operatorname{tr} X^{k}$. If we just keep $N, N_{i}$, and all the combinations $t_{k} / \hbar$ fixed and scale only the formal expansion parameter $\hbar$, we immediately come to (126).

Note that in the case of $1 \mathrm{MM}$ without hard walls, the operator in (126) has also the sense of the scaling transformations under which all vertices are multiplied by the same scaling factor $\rho$ and all the propagators are multiplied by $\rho^{-1}$. The action of derivatives in $t_{0}$ and $S_{i}$ results in multiplying all index loops (faces of the fat graph) by $\rho$. Therefore, for any graph, the total factor is

$$
\rho^{\# \text { vertices - \# edges }+\# \text { faces }}=\rho^{2-2 h} \text {, }
$$

and it is exactly canceled by the scaling of the formal expansion parameter $\hbar \rightarrow \rho \hbar$.

The above relation, being purely kinematic, pertains to any matrix integral irrespectively to the domain of eigenvalues, etc.; to the best of our knowledge, this is the first time when it found a technical application.

On checking the consistency conditions for $h \geq 2$. The consistency condition for $h=0$ are satisfied by construction. We verify these conditions in the case $h=1$ in the next section and consider here only the case $h \geq 2$ in which we have now the explicit representation for the free energy $\mathcal{F}_{h}$.

A simple, but rather naive, consideration pertains to that the whole expression for $\mathcal{F}_{h}$ with $h \geq 2$ can be treated as a functional of $y(\mu)$. Then,

$$
\begin{aligned}
\oint_{\mathcal{C}_{a_{\beta}}} \frac{d \lambda}{2 \pi i}\left[y(\lambda) W_{h}(\lambda)\right]_{-}=\oint_{\mathcal{C}_{a_{\beta}}} \frac{d \lambda}{2 \pi i} y(\lambda) \frac{\partial \mathcal{F}_{h}}{\partial V(\lambda)}=\oint_{\mathcal{C}_{a_{\beta}}} \frac{d \lambda}{2 \pi i} \oint_{\lambda>\mathcal{C}_{\mathcal{D}}} \frac{d \mu}{2 \pi i} y(\lambda) \frac{\partial y(\mu)}{\partial V(\lambda)} \frac{\delta \mathcal{F}_{h}}{\delta y(\mu)} \\
=\oint_{\mathcal{C}_{a_{\beta}}} \frac{d \lambda}{2 \pi i} \oint_{\lambda>\mathcal{C}_{\mathcal{D}}} \frac{d \mu}{2 \pi i} B(\mu, \lambda) y(\lambda) \frac{\delta \mathcal{F}_{h}}{\delta y(\mu)} \\
\frac{\partial \mathcal{F}_{h}}{\partial a_{\beta}}=\oint_{\mathcal{C}_{\mathcal{D}}} \frac{d \mu}{2 \pi i} \frac{\partial y(\mu)}{\partial a_{\beta}} \frac{\delta \mathcal{F}_{h}}{\delta y(\mu)}=\oint_{\mathcal{C}_{a_{\beta}}} \frac{d \lambda}{2 \pi i} \oint_{\lambda<\mathcal{C}_{\mathcal{D}}} \frac{d \mu}{2 \pi i} B(\mu, \lambda) y(\lambda) \frac{\delta \mathcal{F}_{h}}{\delta y(\mu)}
\end{aligned}
$$


and these expressions differ only by the order of integration. ${ }^{9}$

Let us introduce the operator

$$
D_{\beta}=\frac{\partial}{\partial a_{\beta}}-\oint_{\mathcal{C}_{a_{\beta}}} \frac{d \lambda}{2 \pi i} y(\lambda) \frac{\partial}{\partial V(\lambda)} .
$$

When acting on functionals of $y(\mu)$, it can be presented as

$$
\begin{aligned}
D_{\beta} \mathcal{F}_{h}[y] & =\oint_{\mathcal{C}_{a_{\beta}}} \frac{d \lambda}{2 \pi i} \oint_{\lambda<\mathcal{C}_{\mathcal{D}}} \frac{d \mu}{2 \pi i} B(\mu, \lambda) y(\lambda) \frac{\delta \mathcal{F}_{h}}{\delta y(\mu)}-\oint_{\mathcal{C}_{a_{\beta}}} \frac{d \lambda}{2 \pi i} \oint_{\lambda>\mathcal{C}_{\mathcal{D}}} \frac{d \mu}{2 \pi i} B(\mu, \lambda) y(\lambda) \frac{\delta \mathcal{F}_{h}}{\delta y(\mu)}= \\
& =\oint_{\mathcal{C}_{a_{\beta}}} \frac{d \lambda}{2 \pi i} y^{\prime}(\lambda) \frac{\delta \mathcal{F}_{h}}{\delta y(\lambda)},
\end{aligned}
$$

and we can schematically present the action of $D_{\beta}$ as

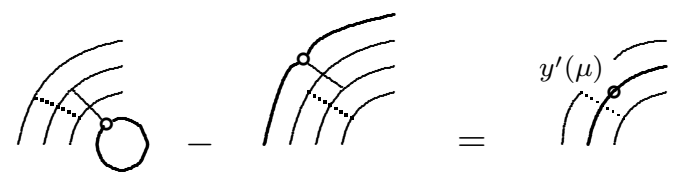

where we let the white dot represent the function $y(\mu)$ and the fat contour designate the integration over the circle about the branching point $a_{\beta}$; apparently, integrations about other branching points commute with this integration. We can now use representation (106), which implies that, actually, when acting on $\mathcal{F}_{h}$, we can put all variations to the outer line to obtain, instead of (131),

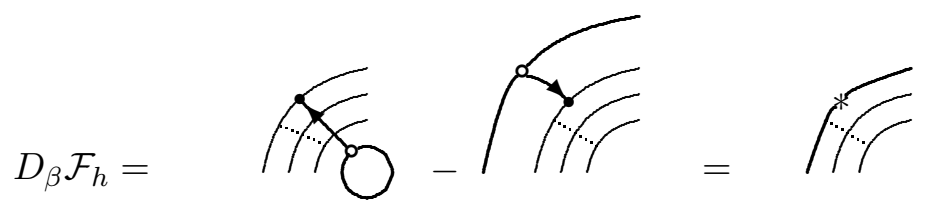

where the asterisk now designates the whole combination $\sum_{h^{\prime}=1}^{h-1} W_{h}^{\prime}(\lambda) W_{h-h^{\prime}}(\lambda)+W_{h-1}(\lambda, \lambda)$ in the r.h.s. of (106). However, for these considerations to work we must be able to collapse integration contours to the branching points to produce representation (109) for the one-loop resolvent. So the above considerations are only plausible reasonings, and in order to prove the consistency conditions rigorously we must use other technique. At present, the only systematic way to prove these conditions is to use the induction in the framework of the above diagrammatic technique. This proof is however too cumbersome to be presented here and will be published elsewhere.

\footnotetext{
${ }^{9}$ We also can substitute just $y(\lambda) W_{h}(\lambda)$ for $\left[y(\lambda) W_{h}(\lambda)\right]_{-}$when evaluating the residue at $\lambda=a_{\beta}$.
} 


\section{Calculation in genus one}

This is the only place where we integrate the expressions for $W_{h}(\lambda)$ explicitly in the spirit of [34] and [35.

\subsection{Finding the free energy}

Choosing a special basis. We now express the action of operator (104) on the monomials $\left(\mu-\mu_{i}\right)$ in terms of the derivatives w.r.t. $\partial / \partial V(\mu)$.

Using the above conditions and formula (90), we can invert the operator $\widehat{K}-2 W_{0}(\lambda)$ when acting on basis monomials $\left(\lambda-\mu_{\alpha}\right)^{-k}$. We define the basis vectors $\chi_{i}^{(k)}(\lambda)$ to be

$$
\chi_{i}^{(k)}(\lambda) d \lambda=\oint_{\mathcal{C}_{\left\{\mu_{j}\right\}}} \frac{d \mu}{2 \pi i} \frac{d \mathcal{E}(\lambda, \mu)}{y(\mu)} \cdot \frac{1}{\left(\mu-\mu_{i}\right)^{k}} \equiv \widehat{d \mathcal{E}}\left(\left(\lambda-\mu_{i}\right)^{-k}\right), \quad i=1, \ldots, 2 n .
$$

Since the r.h.s. of (106) is this operator acting on a collection of monomials, the general form of the one-point resolvent is

$$
W_{h}(\lambda)=\sum_{k=1}^{3 h-1} \sum_{i=1}^{2 n} A_{i, h}^{(k)} \chi_{i}^{(k)}(\lambda), \quad h \geq 1
$$

where $A_{\alpha, h}^{(k)}$ are certain functions of $\mu_{\alpha}, a_{\beta}$, and the moments $M_{i}^{(k)}$. As the order of the highest singularity term $1 /\left(\left(\lambda-\mu_{\alpha}\right)^{3 h-1} \tilde{y}(\lambda)\right)$ in $W_{h}(\lambda)$ is insensitive to a multi-cut structure, $W_{h}(\lambda)$ will depend on at most $2 n(3 h-2)$ moments, just like the one-cut solution 34 . Here we need only few first basis functions.

To obtain $\chi_{\alpha}^{(1)}$, let us start with (80). It implies

$$
\left.\frac{1}{\lambda-\mu_{\alpha}} \frac{\partial \mu_{\alpha}}{\partial V(\mu)}\right|_{\lambda \rightarrow \mu_{\alpha}}=2\left(B(\lambda, \mu)-B\left(\lambda^{*}, \mu\right)\right) \frac{1}{y(\lambda)}+O(1)
$$

whence, as $\int \frac{d \lambda}{\sqrt{\lambda-\mu_{\alpha}}}=2 \sqrt{\lambda-\mu_{\alpha}}$, we have

$$
\left.\frac{\partial \mu_{\alpha}}{\partial V(\mu)}\right|_{\lambda \rightarrow \mu_{\alpha}}=\oint_{\mathcal{C}_{\mu_{\alpha}}} \frac{1}{2 \pi i} \frac{d \mathcal{E}(\mu, \lambda)}{\lambda-\mu_{\alpha}} \frac{1}{y(\lambda)}=\widehat{d \mathcal{E}}\left(\frac{1}{\lambda-\mu_{\alpha}}\right)
$$

It then technically useful to introduce the function

$$
y_{\alpha}(\mu) \equiv y(\mu)\left(\mu-\mu_{\alpha}\right)^{-1 / 2}
$$


which, together with all its derivatives in $\mu$, is nonsingular as $\mu \rightarrow \mu_{\alpha}$. We then apply integration by parts to obtain

$$
\begin{aligned}
& \oint_{\mathcal{C}_{\left\{\mu_{j}\right\}}} \frac{d \mu}{2 \pi i} \frac{d \mathcal{E}(\lambda, \mu)}{y(\mu)} \cdot \frac{1}{\left(\mu-\mu_{\alpha}\right)^{2}}=\oint_{\mathcal{C}_{\mu_{\alpha}}} \frac{d \mu}{2 \pi i} \frac{1}{\mu-\mu_{\alpha}} \frac{\partial}{\partial \mu}\left(\frac{d \mathcal{E}(\lambda, \mu)}{y(\mu)}\right) \\
& =\oint_{\mathcal{C}_{\mu_{\alpha}}} \frac{d \mu}{2 \pi i} \frac{1}{\mu-\mu_{\alpha}}\left(\frac{\left(B(\lambda, \mu)-B\left(\lambda^{*}, \mu\right)\right)}{y(\mu)}+\frac{d \mathcal{E}(\lambda, \mu)}{y(\mu)}\left[-\frac{y_{\alpha}^{\prime}(\mu)}{y_{\alpha}(\mu)}-\frac{1}{2\left(\mu-\mu_{\alpha}\right)}\right]\right) .
\end{aligned}
$$

Taking the term with $\mu-\mu_{\alpha}$ in the square brackets in the r.h.s. to the l.h.s., taking into account that the term with the Bergmann kernel in the r.h.s. is just $-\partial y(\mu) / \partial V(\lambda)$, and using (136), we obtain

$$
\begin{aligned}
\frac{3}{2} & \oint_{\mathcal{C}_{\mu_{\alpha}}} \frac{d \mu}{2 \pi i} \frac{d \mathcal{E}(\lambda, \mu)}{y(\mu)} \cdot \frac{1}{\left(\mu-\mu_{\alpha}\right)^{2}}=\oint_{\mathcal{C}_{\mu_{\alpha}}} \frac{d \mu}{2 \pi i} \frac{1}{\mu-\mu_{\alpha}}\left(-\frac{\partial y(\mu)}{\partial V(\lambda)} \frac{1}{y(\mu)}+\frac{d \mathcal{E}(\lambda, \mu)}{y(\mu)}\left[-\frac{\partial}{\partial \mu} \log y_{\alpha}(\mu)\right]\right) \\
& =\oint_{\mathcal{C}_{\mu_{\alpha}}} \frac{d \mu}{2 \pi i} \frac{1}{\mu-\mu_{\alpha}}\left(-\frac{\partial}{\partial V(\lambda)} \log y_{\alpha}(\mu)+\frac{1}{2\left(\mu-\mu_{\alpha}\right)}\right)-\left.\frac{d \mu_{\alpha}}{\partial V(\lambda)} \frac{\partial}{\partial \mu}\right|_{\mu=\mu_{\alpha}} \log y_{\alpha}(\mu) \\
& =\oint_{\mathcal{C}_{\mu_{\alpha}}} \frac{d \mu}{2 \pi i}\left(-\frac{1}{\mu-\mu_{\alpha}} \frac{\partial}{\partial V(\lambda)} \log y_{\alpha}(\mu)-\frac{d \mu_{\alpha}}{\partial V(\lambda)} \frac{1}{\left(\mu-\mu_{\alpha}\right)^{2}} \log y_{\alpha}(\mu)\right) \\
& =-\frac{\partial}{\partial V(\lambda)}\left(\oint_{\mathcal{C}_{\mu_{\alpha}}} \frac{d \mu}{2 \pi i} \frac{1}{\mu-\mu_{\alpha}} \log y_{\alpha}(\mu)\right) \\
& =-\frac{\partial}{\partial V(\lambda)}\left(\log y_{\alpha}\left(\mu_{\alpha}\right)\right)
\end{aligned}
$$

The last nontrivial action to consider is

$$
\oint_{\mathcal{C}_{\left\{\mu_{j}\right\}}} \frac{d \mu}{2 \pi i} \frac{d \mathcal{E}(\lambda, \mu)}{y(\mu)} \cdot \frac{1}{\left(\mu-a_{\beta}\right)^{2}}=2 \frac{B\left(\lambda,\left[a_{\beta}\right]\right)}{y\left(\left[a_{\beta}\right]\right)} .
$$

On the other hand,

$$
\begin{aligned}
\frac{\partial}{\partial V(\lambda)} M\left(a_{\beta}\right) & =\frac{\partial}{\partial V(\lambda)} \oint_{\mathcal{C}_{a_{\beta}}} \frac{d \mu}{2 \pi i} \frac{y(\mu)}{\mu-a_{\beta}} \frac{1}{\tilde{y}(\mu)} \\
& =\oint_{\mathcal{C}_{a_{\beta}}} \frac{d \mu}{2 \pi i}\left(\frac{-B(\lambda, \mu)}{\mu-a_{\beta}} \frac{1}{\tilde{y}(\mu)}+\frac{M(\mu)}{\mu-a_{\beta}} \sum_{\alpha=1}^{s} \frac{1}{2\left(\mu-\mu_{\alpha}\right)} \cdot \frac{\partial \mu_{\alpha}}{\partial V(\lambda)}\right) \\
& =-\frac{B\left(\lambda,\left[a_{\beta}\right]\right) M\left(a_{\beta}\right)}{y\left(\left[a_{\beta}\right]\right)}+M\left(a_{\beta}\right) \sum_{\alpha=1}^{s} \frac{1}{2\left(a_{\beta}-\mu_{\alpha}\right)} \cdot \frac{\partial \mu_{\alpha}}{\partial V(\lambda)} .
\end{aligned}
$$

Collecting all the results, we have

$$
\chi_{\alpha}^{(1)}(\lambda)=\widehat{d \mathcal{E}}\left(\frac{1}{\lambda-\mu_{\alpha}}\right)=\frac{\partial \mu_{\alpha}}{\partial V(\lambda)}, \quad \alpha=1, \ldots, s,
$$




$$
\begin{aligned}
& \chi_{\alpha}^{(2)}(\lambda)=\widehat{d \mathcal{E}}\left(\frac{1}{\left(\lambda-\mu_{\alpha}\right)^{2}}\right)=-\frac{2}{3} \frac{\partial}{\partial V(\lambda)}\left(\log M_{\alpha}^{(1)} \frac{\prod_{\gamma \neq \alpha}^{s} \sqrt{\mu_{\alpha}-\mu_{\gamma}}}{\prod_{\beta=1}^{t} \sqrt{\mu_{\alpha}-a_{\beta}}}\right), \quad \alpha=1, \ldots, s, \\
& \chi_{\beta}^{(1)}(\lambda)=\widehat{d \mathcal{E}}\left(\frac{1}{\lambda-a_{\beta}}\right)=0, \quad \beta=1, \ldots, t, \\
& \chi_{\beta}^{(2)}(\lambda)=\widehat{d \mathcal{E}}\left(\frac{1}{\left(\lambda-a_{\beta}\right)^{2}}\right)=-2 \frac{\partial}{\partial V(\lambda)}\left(\log M_{\beta}^{(1)} \prod_{\alpha=1}^{s} \sqrt{\mu_{\alpha}-a_{\beta}}\right), \quad \beta=1, \ldots, t .
\end{aligned}
$$

Integrating $W_{1}(\lambda)$. Now we evaluate the action of $\widehat{d \mathcal{E}}$ on $W_{0}(\lambda, \lambda)$ in the special basis above to obtain the subleading term $\mathcal{F}_{1}$ for the free energy. We first obtain the explicit formula for $W_{0}(\lambda, \lambda)$ by differentiating expression (81) w.r.t. $\mu$, subtracting the term $1 /(\lambda-\mu)^{2}$, and taking the limit $\mu \rightarrow \lambda$ :

$$
\begin{aligned}
\frac{\partial}{\partial V(\lambda)} W_{0}(\lambda)= & -\frac{1}{4} \frac{\widehat{y}^{\prime \prime}(\lambda)}{\widehat{y}(\lambda)}-\frac{1}{4} \sum_{i=1}^{n-1} H_{i}(\lambda)\left(\sum_{j=1}^{2 n} \frac{1}{\lambda-\mu_{j}} \oint_{A_{i}} d \xi \frac{1}{\left(\xi-\mu_{j}\right) \widehat{y}(\xi)}\right) \\
= & \frac{1}{16} \sum_{i=1}^{2 n} \frac{1}{\left(\lambda-\mu_{i}\right)^{2}}-\frac{1}{8} \sum_{\substack{i, j=1 \\
i<j}}^{2 n} \frac{1}{\mu_{i}-\mu_{j}}\left(\frac{1}{\lambda-\mu_{i}}-\frac{1}{\lambda-\mu_{j}}\right) \\
& -\frac{1}{4} \sum_{i=1}^{n-1} H_{i}(\lambda)\left(\sum_{j=1}^{2 n} \frac{1}{\lambda-\mu_{j}} \oint_{A_{i}} d \xi \frac{1}{\left(\xi-\mu_{j}\right) \widehat{y}(\xi)}\right) .
\end{aligned}
$$

Turning to the last term containing hyperelliptic integrals, we now prove that

$$
\begin{aligned}
\sum_{i=1}^{n-1} H_{i}\left(\mu_{j}\right) \cdot \oint_{A_{i}} \frac{d \xi}{\left(\xi-\mu_{j}\right) \widehat{y}(\xi)} & =\sum_{i=1}^{n-1} \oint_{A_{i}} \frac{H_{i}(\xi)}{\left(\xi-\mu_{j}\right) \widehat{y}(\xi)} d \xi \\
& =2 \frac{\partial}{\partial \mu_{j}} \log \operatorname{det} \sigma, \quad j=1, \ldots, 2 n
\end{aligned}
$$

where the matrix $\sigma$ has entries

$$
\sigma_{i, k} \equiv \oint_{A_{i}} \frac{\xi^{k-1}}{\widehat{y}(\xi)} d \xi, \quad i, k=1, \ldots, n-1 .
$$

For the canonical polynomials $H_{k}(\xi) \equiv \sum_{l=1}^{n-1} H_{l, k} \xi^{l-1}, \quad k=1, \ldots, n-1$, related to the canonically normalized differentials (156), i.e., such that $\oint_{A_{i}} \frac{H_{k}(\xi)}{\widehat{y}(\xi)} d \xi=\delta_{k, i}$, we obviously have

$$
\sum_{l=1}^{n-1} \sigma_{i, l} H_{l, k}=\delta_{i, k} \quad \text { for } \quad i, k=1, \ldots, n-1 .
$$


Therefore, for all $k>0$ such that $l-k-1 \geq 0$,

$$
\sum_{i=1}^{n-1} H_{l, i} \cdot \oint_{A_{i}} \frac{\xi^{l-k-1}}{\widehat{y}(\xi)} d \xi=0,
$$

and

$$
\sum_{i=1}^{n-1} \oint_{A_{i}} \frac{H_{i}(\xi)-H_{i}\left(\mu_{j}\right)}{\left(\xi-\mu_{j}\right) \widehat{y}(\xi)} d \xi=\sum_{i=1}^{n-1} \oint_{A_{i}} \frac{\sum_{l=2}^{n-1} H_{l, i} \sum_{k=1}^{l-1} \xi^{l-k-1} \mu_{j}^{k-1}}{\widehat{y}(\xi)} d \xi=0,
$$

so we obtain the first part of (144). To obtain the second part, note that by virtue of (146) it is just

$$
\sum_{k=1}^{n-1}\left(2 \frac{\partial}{\partial \mu_{j}} \sigma_{j, k}\right) \sigma_{k, i}^{-1}
$$

so we come to the second equality in (144).

To find $W_{1}(\lambda)$ it remains to act by $\widehat{d \mathcal{E}}$ on (143) using (142). This eventually gives

$$
\begin{aligned}
W_{1}(\lambda)= & \frac{\partial}{\partial V(\lambda)}\left[-\frac{1}{24} \log \prod_{\alpha=1}^{s} M_{\alpha}^{(1)}-\frac{1}{8} \log \prod_{\beta=1}^{t} M_{\beta}^{(1)}-\frac{1}{6} \log \prod_{1 \leq \alpha<\gamma \leq s}\left|\mu_{\alpha}-\mu_{\gamma}\right|\right. \\
& \left.-\frac{1}{6} \log \prod_{\alpha=1}^{s} \prod_{\beta=1}^{t}\left|\mu_{\alpha}-a_{\beta}\right|-\frac{1}{2} \log \operatorname{det} \sigma\right]
\end{aligned}
$$

This expression is easy to integrate. Up to a function $\mathcal{F}\left(\left\{a_{\beta}\right\}\right)$ depending only on $a_{\beta}$, we have

$$
\mathcal{F}_{1}=-\frac{1}{24} \log \left(\prod_{\alpha=1}^{s} M\left(\mu_{\alpha}\right) \cdot \prod_{\beta=1}^{t} M^{3}\left(a_{\beta}\right) \cdot \Delta(\mu)^{4} \cdot \prod_{\alpha, \beta}\left(\mu_{\alpha}-a_{\beta}\right)^{4} \cdot\left(\operatorname{det}_{i, j=1, \ldots, n-1} \sigma_{j, i}\right)^{12}\right),
$$

where $\Delta(\mu)=\prod_{1 \leq \alpha<\gamma \leq s}\left(\mu_{\alpha}-\mu_{\gamma}\right)$ is the Vandermonde determinant. This is our final answer for the genus-one partition function. A natural candidate for a function $\mathcal{F}\left(\left\{a_{\beta}\right\}\right)$ in this case might be a power of the Vandermonde determinant composed from $a_{\beta}$. But, as we demonstrate in the next subsection, explicitly solving the consistency conditions, we obtain $\mathcal{F}\left(\left\{a_{\beta}\right\}\right)=0$ and so result (149) is exact.

\subsection{Verifying the consistency conditions}

Using operator $D_{\beta}$ (129), we can present consistency conditions (108) in the case of $\mathcal{F}_{1}$ in the form

$$
D_{\beta} \mathcal{F}_{1}=\oint_{\mathcal{C}_{a_{\beta}}} \frac{d \xi}{2 \pi i} W_{0}(\xi, \xi)
$$


with $\mathcal{F}_{1}$ from (149) and $W_{0}(\xi, \xi)$ from (143). To obtain the answer we need only to define the action of $D_{\beta}$ on the moments and on the branching point parameters. First, from (82), taking the leading term of $B(\lambda, \mu)$ as $\lambda \rightarrow \mu_{\alpha}$, we immediately obtain

$$
D_{\beta} \mu_{\alpha}=0, \quad \alpha=1, \ldots, s, \quad D_{\beta} a_{\gamma}=\delta_{\beta, \gamma}, \quad \gamma=1, \ldots, t
$$

It is also easy to obtain the action of $D_{\beta}$ on moments $M_{\alpha}^{(k)}$ and $M_{\gamma}^{(k)}$ for $\gamma \neq \beta$. Representing

$$
M_{i}^{(k)}=\oint_{\mathcal{C}_{\mu_{i}}} \frac{d \xi}{2 \pi i} \frac{y(\xi)}{\left(\xi-\mu_{i}\right)^{k} \tilde{y}(\xi)}
$$

in the case $\mu_{i} \neq a_{\beta}$ we obtain that $D_{\beta}$ gives zero when acting on all $\mu_{\alpha}$ in the denominator of the integrand, the contours of integration about $a_{\beta}$ and $\mu_{i}$ do not intersect, so the action of $D_{\beta}$ on $y(\xi)$ also vanishes, and the only contribution comes from the explicit variation of just the parameter $a_{\beta}$, which eventually gives

$$
D_{\beta} M_{i}^{(k)}=-\left.\frac{1}{2} \frac{1}{(k-1) !} \frac{\partial^{k-1}}{\partial \xi^{k-1}}\left(\frac{M(\xi)}{\xi-a_{\beta}}\right)\right|_{\xi=\mu_{i}}, \quad \mu_{i} \neq a_{\beta}
$$

The last quantity to calculate is the variation of $M_{\beta}^{(k)}$. Here, as above, all variations in $\mu_{\alpha}$ disappear, but contribution when varying $y(\xi)$ is nonzero because of contour ordering. Permuting contours of integration as in (131), we obtain extra derivative in $\xi$, so, denoting by $\delta_{a_{\beta}}$ the explicit variation w.r.t. the parameter $a_{\beta}$, we obtain

$$
D_{\beta} M_{\beta}^{(k)}=\oint_{\mathcal{C}_{a_{\beta}}} \frac{d \xi}{2 \pi i} y(\xi)\left(\frac{\partial}{\partial \xi}+\delta_{a_{\beta}}\right) \frac{1}{\left(\xi-a_{\beta}\right)^{k} \tilde{y}(\xi)}=\oint_{\mathcal{C}_{a_{\beta}}} \frac{d \xi}{2 \pi i} \frac{y(\xi)}{\left(\xi-a_{\beta}\right)^{k-1 / 2}} \frac{\partial}{\partial \xi} \frac{1}{\tilde{y}_{\beta}(\xi)}
$$

where, as in (137), we let $\tilde{y}_{\beta}(\xi) \equiv \tilde{y}(\xi) \sqrt{\xi-a_{\beta}}$ denote the regular part of the expression $\tilde{y}(\xi)$ in the local coordinates near $a_{\beta}$. We then eventually obtain

$$
D_{\beta} M_{\beta}^{(k)}=\left.\frac{1}{(k-1) !} \frac{\partial^{k-1}}{\partial \xi^{k-1}}\left(-\frac{1}{2} \sum_{\alpha=1}^{s} \frac{M(\xi)}{\xi-\mu_{\alpha}}+\frac{1}{2} \sum_{\substack{\gamma=1 \\ \gamma \neq \beta}}^{t} \frac{M(\xi)}{\xi-a_{\gamma}}\right)\right|_{\xi=a_{\beta}}, \quad \beta=1, \ldots, t .
$$

Substituting these formulas in (150) and evaluating the both sides of the equation using explicit formulas (149) and (143), we find the exact coincidence, which means that no additional factors depending exclusively on the hard wall parameters $a_{\beta}$ are required to satisfy the consistency conditions. Answer (149) is therefore exact, and we show below that it also agrees with the ideology of Bonnet, David, and Eynard [6]. 


\subsection{Genus-one free energy, determinant representation, and singularities}

We now discuss the result obtained for the genus-one free energy. Comparing it with the "old" matrixmodel two-cut solution [35] we find the coincidence up to the modular transformation that permutes $A$ - and $B$-periods. This is exactly because we set all the $A$-periods (34) of $d S$ (53) constant under the action of $\frac{\partial}{\partial V(\lambda)}$, see (61). On the contrary, if we impose the condition of zero $B$-periods of $d S$, which corresponds to the equal "chemical potentials" $\Pi_{i}$ in different wells of the potential [49, or to additional minimization of free energy (43) w.r.t. occupation numbers (45), ${ }^{10}$ we come to the modularly transformed basis of cycles. In this case, the matrix $\sigma_{i, j}^{(A)}$ of the $A$-periods of $\frac{x^{i} d x}{\widehat{y}(x)}$ must be replaced by the matrix $\sigma^{(B)}$ of the corresponding $B$-periods [17]. Certainly, (149) reproduces the answer of [45, 46] $]^{11}$ for the generic multi-cut solution.

That the only result of interchanging $A$ - and $B$-periods is the interchanging of the corresponding periods in $\sigma$ implies that $e^{\mathcal{F}_{1}}$ is a density, not a function, on the moduli space of curves. Indeed, the corresponding determinants are related as $\operatorname{det} \sigma^{(B)}=\operatorname{det} \tau \operatorname{det} \sigma^{(A)}$, where $\tau_{i, j}=\oint_{B_{i}} d w_{j}$ is the period matrix, which is itself related to $\mathcal{F}_{0}$ (see (57)). In order to compensate this determinant $e^{\mathcal{F}_{1}}$ must be transformed under interchanging $A$ - and $B$-cycles with the additional factor $\left(\operatorname{det} \tau_{i j}\right)^{1 / 2}$, which is character for a section of determinant bundle of the operator $\bar{\partial}$ over the moduli space.

It was proposed in 46 that, in order to match the proper behavior under modular transformations, the operator $\bar{\partial}$ must act on the twisted bosons on the hyperelliptic curves; the factor $e^{\mathcal{F}_{1}}$ is then equal to its determinant. Besides, one also needs to add some corrections due to the star operators [46, 50] that do not contain $\operatorname{det} \sigma$ factors and cannot be restored by the modular invariance alone. These corrections are however necessary to obtain correct result (149).

It seems plausible that we can add arbitrary function of the occupation numbers $S_{i}$ and $t_{0}$ to $\mathcal{F}$ not spoiling the solution to the loop equation. However, if we assume normalizing conditions (89) and (91), which follow from the condition of the "locality" of $\mathcal{F}$, that is, from the condition that $\mathcal{F}$ depends only on local properties of $d S$ near the branching points, we completely fix this ambiguity. The answer then becomes singular as $S_{i} \rightarrow 0$, and this is the singularity we are going to discuss now.

The first type of singular behavior occurs when we shrink a cut with two "dynamical" branching points, e.g., bring $\mu_{2}$ to $\mu_{1}$. Setting $\mu_{2}-\mu_{1}=\epsilon \rightarrow 0$, we first consider what happens to the determinant term when closing the cycle $A_{1}$. We have

$$
\lim _{\mu_{2} \rightarrow \mu_{1}=\mu} \oint_{A_{1}} \frac{\xi^{j-1} d \xi}{\sqrt{\left(\xi-\mu_{1}\right)\left(\xi-\mu_{2}\right) \cdots\left(\xi-\mu_{2 n}\right)}}=\frac{\mu^{j-1}}{\sqrt{\left(\mu-\mu_{3}\right) \cdots\left(\mu-\mu_{2 n}\right)}}
$$

and

$$
\lim _{\mu_{2} \rightarrow \mu_{1}=\mu} \oint_{A_{j}} \frac{\xi^{j-1} d \xi}{\sqrt{\left(\xi-\mu_{1}\right)\left(\xi-\mu_{2}\right) \cdots\left(\xi-\mu_{2 n}\right)}}=\oint_{A_{j}} \frac{\xi^{j-1} d \xi}{(\xi-\mu) \sqrt{\left(\xi-\mu_{3}\right) \cdots\left(\xi-\mu_{2 n}\right)}}, \quad j \neq 1 .
$$

\footnotetext{
${ }^{10}$ This definitely implies the independence (vanishing) of $B$-periods of $d S$ under the action of $\partial / \partial V(\lambda)$.

${ }^{11}$ In [45, the determinant term $\operatorname{det} \sigma$ is omitted from the answer.
} 
Introducing $\widehat{y}_{(n-1)}(\xi)=\sqrt{\left(\xi-\mu_{3}\right) \cdots\left(\xi-\mu_{2 n}\right)}$, we then have for the determinant

$$
\lim _{\mu_{2} \rightarrow \mu_{1}=\mu} \sigma_{(n)}=\frac{1}{\sqrt{\left(\mu-\mu_{3}\right) \cdots\left(\mu-\mu_{2 n}\right)}} \cdot \operatorname{det}\left(\begin{array}{ccccc}
1 & \oint_{A_{2}} \frac{d \xi}{(\xi-\mu) \widehat{y}_{(n-1)}(\xi)} & \cdots & \oint_{A_{g}} \frac{d \xi}{(\xi-\mu) \widehat{y}_{(n-1)}(\xi)} \\
\mu & \oint_{A_{2}} \frac{\xi d \xi}{(\xi-\mu) \widehat{y_{(n-1)}(\xi)}} & \cdots & \oint_{A_{g}} \frac{\xi d \xi}{(\xi-\mu) \widehat{y}_{(n-1)}(\xi)} \\
\vdots & \vdots & \ddots & \vdots \\
\mu^{n-2} & \oint_{A_{2}} \frac{\xi^{n-2} d \xi}{(\xi-\mu) \widehat{y}_{(n-1)}(\xi)} & \cdots & \oint_{A_{g}} \frac{\xi^{n-2} d \xi}{(\xi-\mu) \widehat{y}_{(n-1)}(\xi)}
\end{array}\right)
$$

and subtracting now the first column multiplied by $\oint_{A_{k}} \frac{d \xi}{(\xi-\mu) \widehat{y}_{(n-1)}(\xi)}$ from the $k$ th column for all $k=2, \ldots, g$, we obtain just the determinant of $\sigma_{(n-1)}$ with the prefactor $1 / \widehat{y}_{(n-1)}(\mu)$ :

$$
\lim _{\mu_{2} \rightarrow \mu_{1}=\mu} \operatorname{det} \sigma_{(n)}=\frac{1}{\sqrt{\left(\mu-\mu_{3}\right) \cdots\left(\mu-\mu_{2 n}\right)}} \operatorname{det} \sigma_{(n-1)} .
$$

If we denote $\mu_{1}-\mu_{2}=\epsilon$, then in the limit as $\mu_{1} \rightarrow \mu_{2}=\mu, S_{1}=\epsilon^{2} \tilde{y}_{(n-1)}(\mu) M(\mu)$ and the products of branching points are then combined exactly in a way to give

$$
\lim _{\mu_{2} \rightarrow \mu_{1}=\mu} \mathcal{F}_{1}^{(n)}=\mathcal{F}_{1}^{(n-1)}-\frac{1}{12} \log S_{1}+O(\epsilon),
$$

where we must substitute in the expression for $\mathcal{F}_{1}^{(n-1)}$ the new function $\bar{M}(\xi)=M(\xi)(\xi-\mu)$ with the new zero at $\mu$ obtained by colliding the branching points $\mu_{1}$ and $\mu_{2}$.

The singular part of $\mathcal{F}_{1}$ as $S_{i} \rightarrow 0$ has therefore the structure

$$
\left(\mathcal{F}_{1}\right)_{\operatorname{sing}}=-\frac{1}{12} \sum_{i=1}^{n} \log S_{i}, \quad S_{n} \equiv t_{0}-\sum_{j=1}^{n-1} S_{j} .
$$

This is, in fact, a general phenomenon in the matrix model calculations: at any order of 't Hooft expansion, the only potential source for the singular contribution comes from degenerate geometry of curves, and it is related with the normalization factor in the matrix integral, that is, with the volume of (the orbit of) the unitary (sub)group. Indeed, the integral itself is a Taylor series in $S_{i}$ 's (see formula (4.8) in [17]), while the unitary group volume [51] in the case of Hermite polynomial distribution contributes the factor

$$
\int_{-\infty}^{+\infty} \prod_{k=1}^{S_{i} / \hbar} d \lambda_{k} \Delta^{2}(\lambda) e^{-\frac{t_{2}}{2 \hbar} \sum_{k=1}^{S_{i} / \hbar} \lambda_{k}^{2}}=\left(\frac{\hbar}{t_{2}}\right)^{\frac{S_{i}^{2}}{2 \hbar^{2}}} \prod_{k=1}^{S_{i} / \hbar} \Gamma(k) \equiv e^{\frac{S_{i}^{2}}{2 \hbar^{2}} \log \left(\hbar / t_{2}\right)} G_{2}\left(S_{i} / \hbar\right),
$$

with $G_{2}(x)$ being the Barnes function [52] whose asymptotic expansion as $x \rightarrow \infty$ reads [52, 53]

$$
\log G_{2}(x)=\frac{x^{2}}{2} \log x-\frac{1}{12} \log x-\frac{3}{4} x^{2}+\frac{1}{2} x \log 2 \pi+\zeta^{\prime}(-1)+\sum_{h=2} \frac{B_{2 h}}{4 h(h-1)} \frac{1}{x^{2 h-2}},
$$


where $B_{2 h}$ are Bernoulli coefficients and $\zeta(s)$ is the Riemann $\zeta$-function. Discarding all polynomial terms, we obtain that the singular contribution as $S_{i} \rightarrow 0$ is [17]

$$
\left(\mathcal{F}_{0}\right)_{\text {sing }}=\frac{S_{i}^{2}}{2} \log S_{i}, \quad\left(\mathcal{F}_{1}\right)_{\text {sing }}=-\frac{1}{12} \log S_{i}, \quad\left(\mathcal{F}_{h}\right)_{\operatorname{sing}}=\frac{B_{2 h}}{4 h(h-1)} \frac{1}{S_{i}^{2 h-2}}, \quad h \geq 2,
$$

in the case where two dynamical branching points collide. This is the simplest way to segregate singular contribution; for instance, direct calculations demonstrate that the answer for genus-two correction in the one-cut case [34] has the prescribed singularity $-\frac{1}{240} \frac{1}{t_{0}^{2}} \cdot{ }^{12}$

In the hard-wall case, we also have the singularity appearing when a dynamical point collides with the hard wall, $\mu_{1} \rightarrow a_{1}$. In this case, we must calculate the asymptotic expressions for Laguerre, not Hermite, polynomials as the characteristic expression in this case is

$$
\int_{0}^{+\infty} \prod_{k=1}^{S_{i} / \hbar} d \lambda_{k} \Delta^{2}(\lambda) e^{-\frac{t_{1}}{\hbar} \sum_{k=1}^{S_{i} / \hbar} \lambda_{k}}=\left(\frac{\hbar}{t_{1}}\right)^{\frac{S_{i}^{2}}{\hbar^{2}}} \prod_{k=1}^{S_{i} / \hbar}(\Gamma(k))^{2} \equiv e^{\frac{S_{i}^{2}}{\hbar^{2}} \log \left(\hbar / t_{1}\right)}\left(G_{2}\left(S_{i} / \hbar\right)\right)^{2},
$$

because the norm $h_{k}$ for the normalized Laguerre polynomial $\frac{\partial^{n}}{d x^{n}}\left(x^{n} e^{-x}\right)$ is $(n !)^{2}$. Then exactly the same considerations yield the corresponding singularities:

$$
\left(\mathcal{F}_{0}\right)_{\text {sing }}=S_{i}^{2} \log S_{i}, \quad\left(\mathcal{F}_{1}\right)_{\operatorname{sing}}=-\frac{1}{6} \log S_{i}, \quad\left(\mathcal{F}_{h}\right)_{\operatorname{sing}}=\frac{2 B_{2 h}}{4 h(h-1)} \frac{1}{S_{i}^{2 h-2}}, \quad h \geq 2 .
$$

Comparing with result (149) using the same considerations as above (in particular, the behavior of $\operatorname{det} \sigma$ is exactly the same), we find that

$$
\lim _{\mu_{1} \rightarrow a_{1}=\mu} \mathcal{F}_{1}^{(n)}=\mathcal{F}_{1}^{(n-1)}-\frac{1}{6} \log S_{1}+O(\epsilon),
$$

where $S_{1} \sim \epsilon M(\mu) \tilde{y}_{(n-1)}(\mu)$ and the function $M(\xi)$ remains unchanged because no new zeros or poles arise when $\mu_{1} \rightarrow a_{1}$. This demonstrates again the consistency of result (149) because the presence of additional factors depending on parameters $a_{\beta}$ in $\mathcal{F}_{1}$ would affect the (nonsingular) part of relation (162).

\section{Conclusion}

In conclusion, we briefly mention some related papers. Perhaps the first consideration of the WDVV equations for the differential $d S$ with square-root singularities at the denominator was done from the matrix-model standpoint by Itoyama and Morozov [54. There, it was used to regularize the matrixmodel integral. Worth mentioning is also recent paper [55] in which the CFT technique has been used

\footnotetext{
${ }^{12}$ In the proper normalization in which $t_{0}=d^{2} / 16$ for $M(\xi) \equiv 1$.
} 
for finding multiresolvents for the simplest, one-cut zero-potential matrix model with two hard edges. The asymptotic expansion for such an integral (the Legendre polynomials) reads

$$
\begin{array}{r}
\log \int_{-a}^{a} \Delta^{2}(\lambda) \prod_{k=1}^{t_{0} / \hbar} d \lambda_{k}=\frac{t_{0}^{2}}{\hbar^{2}} \log (a / 8)+\frac{t_{0}}{\hbar} \log 2 \pi+3 \zeta^{\prime}(-1)-\frac{1}{4} \log \left(t_{0} / \hbar\right) \\
+\frac{1}{12} \log 2+\sum_{h=2}^{\infty}\left(\frac{\hbar}{t_{0}}\right)^{2 h-2} \frac{B_{2 h}}{h(h-1)}\left(1-\frac{1}{2^{2 h}}\right)
\end{array}
$$

and in particular we see that the third derivative of the leading term vanishes, as follows from (97).

\section{Acknowledgments}

The author is grateful to his collaborators B. Eynard, A. Marshakov, A. Mironov, and D. Vassiliev with whom a part of results of this paper was obtained. He also acknowledges the useful discussion with M. Bertola, D. Korotkin, and J. Harnad.

This work has been partially financially supported by the RFBR Grant No. 05-01-00498, Grants of Support for the Scientific Schools 2052.2003.1, by the Program Mathematical Methods of Nonlinear Dynamics, by the ANS Grant "Géométrie et Intégrabilité en Physique Mathématique" (contract number ANR-05-BLAN-0029-01), and by the European Community through the FP6 Marie Curie RTN ENIGMA (Contract number MRTN-CT-2004-5652).

\section{References}

[1] F.Cachazo, K.Intriligator, and C.Vafa, Nucl.Phys. B603 (2001) 3-41, hep-th/0103067

[2] F.Cachazo and C.Vafa, hep-th/0206017

[3] R.Dijkgraaf and C.Vafa, Nucl.Phys. 644 (2002) 3-20, hep-th/0206255 Nucl.Phys. 644 (2002) 21-39, hep-th/0207106; hep-th/0208048

[4] K.Demeterfi, N.Deo, S.Jain and C.-I Tan, Phys.Rev. D42 (1990) 4105-4122

J.Jurkiewicz, Phys.Lett. 245 (1990) 178

C̆.Crnković and G.Moore, Phys.Lett. B257 (1991) 322

[5] G.Akemann and J.Ambjørn, J.Phys. A29 (1996) L555-L560, cond-mat/9606129

[6] G.Bonnet, F.David, and B.Eynard, J.Phys. A33 (2000) 6739-6768

[7] I.Krichever, Commun.Pure Appl.Math. 47 (1992) 437; hep-th/9205110 
[8] A.Marshakov and A.Mironov, hep-th/9809196

[9] A.Marshakov, Seiberg-Witten Theory and Integrable Systems, World Scientific 1999.

[10] H.W.Braden and I.M.Krichever (Eds.), Integrability: The Seiberg-Witten and Whitham Equations, Gordon and Breach, 2000

[11] A.Gorsky and A.Mironov, hep-th/0011197

[12] L.Chekhov and A.Mironov Phys.Lett. 552B (2003) 293-302, hep-th/0209085

[13] V. A. Kazakov and A. Marshakov, J. Phys. A 36 (2003) 3107, hep-th/0211236

[14] A.Gerasimov, A.Marshakov, A.Mironov, A.Morozov and A.Orlov, Nucl.Phys. B357 (1991) 565618

S.Kharchev, A.Marshakov, A.Mironov, A.Orlov and A.Zabrodin, Nucl.Phys. B366 (1991) 569601 ;

A.Morozov, Phys.Usp. 37 (1994) 1-55; hep-th/9502091

[15] A.A. Migdal, Phys.Rep. 102 (1983) 199

J. Ambjørn, J. Jurkiewicz and Yu. Makeenko, Phys.Lett. B251 (1990) 517

Yu. Makeenko, Mod.Phys.Lett. (Brief Reviews) A6 (1991) 1901-1913

[16] F.David, Mod.Phys.Lett. A5 (1990) 1019

A.Mironov and A.Morozov, Phys.Lett. B252 (1990) 47-52

Ambjørn J. and Makeenko Yu., Mod.Phys.Lett. A5 (1990) 1753

H.Itoyama and Y.Matsuo, Phys.Lett. 255B (1991) 202

[17] A.Klemm, M.Marino, and S.Theisen, JHEP 0303 (2003) 051, hep-th/0211216

[18] A.Alexandrov, A.Mironov and A.Morozov, Int.J.Mod.Phys. A19 (2004) 4127-4165; hep-th/0310113

[19] A.Alexandrov, A.Mironov and A.Morozov, Fortschritte der Physik, 53 (2005) 512, hep-th/0412205

[20] L.Chekhov, A.Marshakov, A.Mironov, and D.Vasiliev, Phys. Lett. 562B (2003) 323-338, hep-th/0301071

[21] L.Chekhov, A.Marshakov, A.Mironov, and D.Vasiliev, Proc. Steklov Math. Inst. 251 (2005) 254-292; hep-th/0506075

[22] B.Eynard, JHEP 0411:031 (2004); hep-th/0407261

[23] L.Chekhov and B.Eynard, hep-th/0504116 
[24] G. Szegö, Orthogonal Polynomials, AMS Publ., Providence, R.I. 1981 (4th edn.).

[25] B. Eynard, math-ph/0504002.

[26] M. Bertola, hep-th/0511295.

[27] E.Witten, Nucl.Phys. B340 (1990) 281-332

[28] R.Dijkgraaf, E.Verlinde, and H.Verlinde, Nucl.Phys. B352 (1991) 59-86

[29] A.Marshakov, A.Mironov and A.Morozov, Phys.Lett. B389 (1996) 43, hep-th/9607109

[30] A.Marshakov, A.Mironov and A.Morozov, Mod.Phys.Lett. A12 (1997) 773-787, hepth/9701014

[31] M. Bertola, JHEP 0311:062 (2003); hep-th/0309192.

[32] B. Eynard, A. Kokotov, and D. Korotkin, Nucl.Phys. B694 (2004) 443-472; hepth/0403072.

[33] M. Adler and P. van Moerbeke, Ann. Math. 153 (2001) 149-189; math-ph/0009001.

[34] J.Ambjørn, L.Chekhov, C.F.Kristjansen and Yu.Makeenko, Nucl.Phys. B404 (1993) 127-172; Erratum ibid. B449 (1995) 681, hep-th/9302014

[35] G.Akemann, Nucl.Phys. B482 (1996) 403, hep-th/9606004

[36] B. de Wit and A. Marshakov, Theor. Math. Phys. 129 (2001) 1504 [Teor. Mat. Fiz. 129 (2001) 230], hep-th/0105289

[37] N.Seiberg and E.Witten, Nucl.Phys. B426 (1994) 19-52; [Erratum: ibid. B430 (1994) 485], hep-th/9407087; Nucl.Phys., B431 (1994) 484-550.

[38] H.W.Braden and A.Marshakov, Nucl.Phys. B595 (2001) 417-466, hep-th/0009060

[39] A.Gorsky, I.Krichever, A.Marshakov, A.Mironov and A.Morozov, Phys.Lett. B355 (1995) 466474

[40] A.Gorsky, A.Marshakov, A.Mironov and A.Morozov, Nucl.Phys. B527 (1998) 690-716

[41] J.Fay, Theta-Functions on Riemann Surfaces, Lect.Notes Math., Vol. 352, Springer, New York, 1973.

[42] H.E. Rauch, Comm. Pure Appl. Math. 12 (1959) 543-560.

[43] A.Boyarsky, A.Marshakov, O.Ruchayskiy, P.Wiegmann and A.Zabrodin, Phys.Lett. 515B (2001) 483-492, hep-th/0105260

[44] A. Marshakov, hep-th/0108023 Theor.Math.Phys. 132 (2002) 895, hep-th/0201267 
[45] I.K.Kostov, hep-th/9907060

[46] R.Dijkgraaf, A.Sinkovics and M.Temürhan, hep-th/0211241

[47] Al. Zamolodchikov, Nucl.Phys. B285 [FS19] (1987) 481

[48] L.Chekhov, Theor.Math.Phys. 141 (2004) 1640-1653, hep-th/0401089

[49] F.David, Phys.Lett. B302 (1993) 403-410, hep-th/9212106

O.Lechtenfeld, Int.J.Mod.Phys. A7 (1992) 2335

[50] G. W. Moore, in: Proc. Cargese meeting on Random Surfaces, Quantum Gravity, and Strings, 1990, Prog. Theor. Phys. Suppl. 102 (1990) 255

[51] S.Kharchev, A.Marshakov, A.Mironov and A.Morozov, Nucl.Phys. B397 (1993) 339, hep-th/9203043

[52] E.W.Barnes, Phil.Trans.Roy.Soc. A196 (1901) 265-387

[53] See also Appendix A for a compressed list of results on the Barns functions in:

M.Jimbo and T.Miwa, J.Phys. A29 (1996) 2923-2958, hep-th/9601135

[54] H.Itoyama and A.Morozov, Nucl. Phys. B657 (2003) 53-78; hep-th/0211245

[55] M.R. Gaberdiel, A.Klemm, and I.Runkel hep-th/0509040 